\title{
Closely Coupled Fluid-Solid Interface Method with Moving-Average for LES based Conjugate Heat Transfer Solution
}

\author{
L. He \\ Department of Engineering Science, \\ University of Oxford
}

\begin{abstract}
Fluid-solid coupled Conjugate Heat Transfer (CHT) simulations are relevant to many practical problems. Most existing interfacing methods have been developed for Reynolds averaged Navier-Stokes solvers. For high fidelity turbulence scale-resolved flow solvers however, the CHT interface methods face significant challenges arisen from a wide frequency spectrum of unsteady disturbances to be dealt with, compounded by the huge time scale disparity between fluid and solid domains.

In this paper, a closely coupled non-partitioned (monolithic) CHT method is presented. The main issues of interest are the prohibitive time costs of direct time domain CHT methods and an extra mesh dependency in the solid domain when resolving high frequency turbulence disturbances. Based on a temporal Fourier spectral framework, the present CHT interface method entails a moving-average for the time-mean flow and a discrete Fourier transform on-the-fly at each time step. Taking advantage of a semi-analytical transfer function and harmonic balancing for the CHT interface, we can achieve solving the solid domain completely in its own time step (3-5 orders of magnitude larger than that of the fluid domain). The present interface method can effectively circumvent aliasing errors and extra solid domain mesh-dependence encountered by other time-domain coupling methods when applied to turbulence scale-resolved CHT solutions. Illustrative stability analyses also show that the numerical stability of the present CHT interface should require no more stringent conditions than that in either fluid or solid domain. The computational results and analyses highlight the advantages of the present methodology in terms of both the computational efficiency and accuracy, in comparison with a conventional directly coupled interface method. Furthermore, a case study aided by a simple interface response analysis highlights much augmented wall temperature fluctuations and higher sensitivity to the interface treatment when a low conductivity protection layer (Thermal Barrier Coating, TBC) is added. The present study underlines the relevance of accounting for fluid disturbances over a range of frequencies in an effective and accurate CHT interface treatment.
\end{abstract}

\section{Keywords:}

Conjugate Heat Transfer; Fluid-Solid Interface; Large Eddy Simulation; Fourier Method.

\section{Introduction}

Prediction of convective heat transfer is relevant to a wide range of engineering problems, especially in relation to the performance, thermal mechanical integrity and lifespan of hot-end components of gas turbines/aero-engines. Conventional methods for convective heat transfer prediction have been predominantly based on the use of heat transfer coefficient (HTC). The 
fundamental assumption of a HTC based method is that convective heat transfer is predominantly determined by fluid mechanics/aerodynamics with negligible feedback from heat transfer at a fluid-solid boundary to the flow, which is consistent with the linear relation between heat flux and wall temperature, as implied in the Newton's law of cooling. Early methods for convective heat transfer prediction are commonly based on empirical correlations. In the past few decades, development of computational fluids dynamics (CFD) has greatly enhanced capabilities in predicting detailed flow field features, which have in turn enhanced the prediction of detailed HTC distributions in a complex flow field.

It has been argued that the validity and applicability of HTC based approaches may be questionable under some conditions, e.g. Moffat (1998). For forced convection around high pressure turbine blades at realistic gas-wall temperature ratios, there are indications that HTC can be dependent on the temperature ratio, e.g. Maffulli and He, (2014), (2017), Zhang and He, (2014). The noted dependency of HTC on wall temperatures provides some justifications for development of fluid-solid coupled conjugate heat transfer (CHT) methods. For natural convection problems, the close inter-dependence between a solid wall and its adjacent thermally driven fluid flow presents a stronger challenge to a HTC based approach, thus a stronger case for CHT methods.

When developing a fluid-solid coupled CHT, a fundamental issue one has to face is the time-scale disparity between fluid and solid domains. For common aerothermal problems of gas turbine blades, a typical time scale in a solid domain can be by $10^{4}$ longer than that in a fluid domain (He and Oldfield, 2011). For steady flows, this time-scale disparity is not a problem for CHT solutions as a converged solution does not need to respect the physical time history. Also the domain-dependent local time stepping scheme can be easily implemented in widely used time-marching solvers to drive to a steady state solution very effectively. The CHT solutions however become very acute for unsteady flow problems. The physical time step needs to be small enough to provide a sufficient temporal resolution for the fast changing fluid part. But on the other hand, the temporal time scale to be covered (marched) for a time-accurate CHT needs to be sufficiently long for the thermal disturbances to be propagated through the solid domain. For turbine blade heat transfer and cooling studies, there have been clear evidences to support use of turbulence scale-resolved large eddy simulations (LES) types of high fidelity models, computationally and experimentally, e.g. Tyagi and Acharya (2003), Fawcett et al (2013). The challenge arisen from the conflicting requirements due to the time scale disparity for unsteady CHT problems is further augmented when a higher fidelity turbulence scale resolved flow solver, LES or direct numerical simulations (DNS), is adopted, for which even smaller time step sizes are required. In general, it is noted that there has been considerable recent advancement in adopting higher fidelity flow models for convective heat transfer predictions and simulations. On the other hand, the development of the related fluid-solid interface treatment seems lagging behind, despite the increased challenges due to the hugely enlarged time scale disparity when a turbulence scale resolved flow model is adopted.

A directly coupled interface treatment can be easily realised, to simultaneously satisfy both the temperature and heat flux continuity, Perelmans (1961). Similarly to the field solvers for the fluid and solid domains, the directly coupled interface method works stably and effectively for steady flows when very different time steps, e.g. those dictated by the numerical stability for explicit schemes, are used in the two domains respectively and the time consistency 
and accuracy are not of concern. However, the directly coupled and time accurate CHT solution (i.e. completely time-synchronised in both the fluid and solid domains) are prohibitively timeconsuming due to the required time consistence for an unsteady problem. This may be the chief reason that many previous CHT methods are based on partitioned CHT solvers where the two domains are solved by separate fluid flow and solid conduction solvers which are iteratively (sequentially) coupled at the interface (thus this kind of 'partitioned' methods may also be called 'loosely coupled'). In a typical loosely coupled CHT (e.g. Sun et al, 2010), a steady or unsteady RANS fluid solver is coupled with a solid conduction solver. Considerable recent efforts in developing interfacing methods have been made for partitioned loosely coupled CHT methods, e.g. Errera and Baqué (2013), Verstraete and Scholl (2016). When a high fidelity unsteady flow solver is adopted, the time-averaged flow solution will communicate with the corresponding solid solution at the interface. The loosely coupled CHT methods are also developed and applied with LES flow solvers to forced convection, e.g. Duchaine et al (2009), and natural convection problems, e.g. Fadl and He (2017).

It is of the present interest to explore a non-partitioned CHT with an unsteady turbulence scale-resolved flow solver. In a non-partitioned (monolithic) CHT approach, the fluid and solid domain can be discretised and solved using the same solution method. The interface treatment thus tends to be much simpler, e.g. Tiselj et al (2013) in which a DNS solver of an incompressible flow model is applied to a non-partitioned CHT solution. Another tangible advantage of a monolithic CHT method is that there is no need to exchange boundary data between two solvers, which can be a substantial task when there is a large amount of boundary data for multiple and large fluid-solid interfacing boundaries, e.g. in a porous medium or similar microstructures with complex and large fluid-solid interfaces. The locality of the coupling data dependence of a monolithic CHT also makes it much easier for exploiting parallel processing, which can be nontrivial (e.g. Jaure et al 2013).

In relation to recent unsteady time-domain CHT method developments with LES as the flow solvers, attention is drawn to two specific issues relevant to the CHT solution accuracy and consistency.

Firstly, to accelerate an unsteady CHT solution, a time desynchronization can be adopted in which the two domains can be solved in different time step sizes and coupled at different time instants. It has been shown however that a large time-desynchronization may lead to notable aliasing errors (Jaure et al 2013).

Secondly, a directly coupled CHT solution is also shown to be subject to an extra mesh dependency in the solid domain, when a turbulence scale resolved solver is used, as indicated in a LES combustor CHT simulation by Shahi et al (2014). In the context of LES-CHT solutions for steam turbine configurations subject to natural convection, Fadl and He (2017) observed a similar behaviour, and further analysed the wall temperature response to a fluid temperature perturbation. The corresponding errors in the wall temperature and flux fluctuations are shown to be caused by the conflict between the thermal penetration depth being much smaller than a near wall solid mesh size and the required energy balance within a combined fluid and solid mesh cell, Fadl and He (2017). The solid mesh dependency can be acute for LES based CHT, given the wide range of flow disturbance frequencies (thus penetration depths) involved.

It is noted that a two-scale time-decomposition approach has been proposed by $\mathrm{He}$ and Fadl (2016) to address the basic time scale disparity issue for general transient CHT solutions. 
A similar time-decomposition has been adopted by Koren et al (2018) to accelerate CHT problems solved with a LES flow solver. Although the time decomposition should be effective in propagating initial numerical transients in the solid domain as intended, it is questionable that such time-decomposition by itself would help address the two specific issues of interest (the aliasing due to the time-desynchronization and the extra solid domain mesh-dependency) if the short scale unsteady part is still solved in the time domain as in Koren et al (2018).

The above review and observations point to two preferred features in LES based CHT developments:

1) No partitioned computational domains will be required so that no data transfer between solvers during a CHT solution will be entailed, and the fluid-solid interface is treated completely locally.

2) The interface method can effectively deal with the difficulties caused by the solidfluid time scale disparity particularly acute for a turbulence scale resolved flow solvers, and do so accurately and stably. More specifically, the method should be free of the aliasing errors and the extra mesh dependency in the solid domain.

In relation to 2) above. Two specific desired attributes are further identified:

a) The fluid side of the interface should be sampled at each and every time step, so that no high frequency disturbances will be missed or given a chance to alias.

b) The solid side should avoid responding to high frequency flow disturbances, so that the solid conduction solution will only need to capture the transients of long time scales of the solid domain, completely independent of the high frequency turbulence spectrum. When the overall boundary conditions are steady and the only source of the unsteadiness is turbulence, the solid domain can then be solved by its own maximum local time step subject only to the numerical stability.

The present development of a moving-average based CHT interface method is aimed to meet a) and b) above. The basic approach is to realign the mismatched time scales in the fluid and solid domains by converting an unsteady problem to a set of 'steady-like' problems. The time-mean state CHT interfacing can be effectively obtained by a simple moving timeaveraging, thus enabling for the two sides to be coupled in an equivalently steady manner. The basic framework has been proposed for deterministic periodic unsteadiness by He and Oldfield (2011). The main interest of the present work on the other hand is in LES-based CHT interface. With a Fourier spectral representation of the unsteady part, the unsteady CHT coupling can be conducted by harmonic balancing at the interface through a new semi-analytical transfer function. As such, neither a time-accurate fluid-solid interface, nor an unsteady conduction solution in the solution domain would be needed. The solid domain can thus be solved with its time step being completely dictated by the numerical stability, leading to a solid time step size typically by 3-5 orders of magnitude larger than the time step for a corresponding LES flow solution.

In the following sections, the monolithic discretization and solution method as applied to both the fluid and solid domains will be introduced first. Then the non-partitioned CHT system will be described, with the stability of the interface methods examined by a simplified analysis for certain conditions. Finally the impact of the interface methods on the accuracy of the LES based CHT solutions is examined and illustrated. 


\section{Solution Method for Fluid and Solid Models in CHT System}

As introduced, we intend to use a single solution method for a computational domain with fluid and solid sub-domains. The governing equations for either a fluid or solid domain can be written in a simple form:

$$
\frac{\partial \boldsymbol{U}}{\partial t}=R(\boldsymbol{U})
$$

A general framework for transient CHT with two distinctive time scales is presented by He and Fadl (2016), which was implemented and demonstrated for a loosely coupled (partitioned) CHT for natural convection. It is based on decomposing a fluid variable to a long (macro) time scale component and the short (micro) scale one.

$$
\boldsymbol{U}(x, t)=\widetilde{\boldsymbol{U}}(x, t)+\boldsymbol{U}^{\prime}(x, t)
$$

Although the present method can be more generally formulated in the two-scale transient CHT framework, it is decided to confine the formulations to a steady CHT problem for clarity and simplicity. In the context of LES, the fluid domain is naturally unsteady. A steady CHT problem in the present LES context should further be specified as the one where a timeaveraged state exists. Then a variable can be simply decomposed into its time-averaged component and unsteady fluctuation:

$$
\boldsymbol{U}(x, t)=\widetilde{\boldsymbol{U}}(x)+\boldsymbol{U}^{\prime}(x, t)
$$

\subsection{Solution Method and Unsteady Flow Model}

The solution method is based on an in-house multi-block flow code which the author has developed and updated over many years, He (1993), (2008), (2017).

The baseline flow model is the 3-dimensional unsteady Reynolds averaged NavierStokes equations. The computational domain is discretised with a multi-block structured mesh. The equations are spatially discretized using a cell centre based finite volume scheme. The spatial discretization for the advective fluxes is based on the pressure-convective flux split upwinding discretization, AUSM, Liou (2006). The baseline implementation of the AUSM is 2nd order accurate in space for a uniformly regular mesh, following van Leer's MUSCL formulation. A relatively simple extension to a $3^{\text {rd }}$ order spatial accuracy has been adopted, following Cada and Torrilhon (2009), through a suitable reformulation of the nonlinear limiter. This $3^{\text {rd }}$ order scheme has a compact stencil formulation for the limiter. For a flux calculation on a mesh cell face, only should the variables from its 4 adjacent mesh cells be needed. The viscous dissipation terms are discretised using the $2^{\text {nd }}$ order central difference as applied in a finite volume scheme to a mesh cell.

The inlet/exit far-field boundary conditions can be given by the compatibility conditions: specification of the inlet stagnation pressure and temperature, the inflow angle, and the exit static pressure. Alternatively, the local characteristics based nonreflective boundary conditions can be used. On a solid surface, a nonslip wall condition is used for a viscous wall boundary, or a symmetry condition for an inviscid wall boundary. 
The spatially discretised equations are explicitly integrated in time by a 4-stage RungeKutta scheme. For unsteady flows with low frequency disturbances, the physical temporal terms are discretized by a $2^{\text {nd }}$ order backward difference in form of a source term, and the 4stage Runge-Kutta scheme is used to march in pseudo time to converge the solution at each physical time step. The pseudo time marching (sub-iterations) can be accelerated by local time stepping and multigrid as for steady flow solutions. For the present LES solution however, the explicit 4-stage Runge-Kutta scheme is directly adopted in the integration in the physical time to resolve high frequency turbulence disturbances.

The semi-discretised flow equation for stage $\mathrm{k}$ of the 4-stage Runge-Kutta integration at time step m can be simply expressed as,

$$
\boldsymbol{U}_{m}^{k}=\boldsymbol{U}_{m-1}+c_{k} \Delta t_{f} R_{f}\left(\boldsymbol{U}_{m}^{k-1}\right)
$$

where the coefficients for the 4-stage Runge-Kutta scheme of $2^{\text {nd }}$ order accuracy are:

$$
c_{k}=\frac{1}{5-k}, \quad(k=1,2,3,4)
$$

And $\Delta t_{f}$ denotes the fluid time step size. The time-step size is limited by the minimum mesh spacing as required by the numerical stability, the usable time step can be augmented for non-uniform mesh spacing cases by using a time-consistent multi-grid, He (1993).

The closure of turbulence stress terms for RANS solutions is made with two options: the one-equation Spalart-Allmarus model, and the k- $\omega$ SST two-equation model. Large eddy simulations (LES) are performed with a Smagorinsky model for closing the sub-grid scales.

\subsection{Solid Conduction Model}

For the solid domain, the temperature field is governed by the unsteady heat conduction equation effectively in the same form as the fluid energy equation, with zero velocities and corresponding material properties. The equation is in a similar simple form to those for the fluid domain:

$$
\frac{\partial T_{S}}{\partial t}-R_{S}\left(T_{S}\right)=0
$$

The solid domain is solved with the same discretization and integration method as for the fluid domain, as a monolithic CHT method is meant to be. Similar to the viscous dissipation terms for the fluid equation, the energy equation for the solid domain is discretised using the same $2^{\text {nd }}$ order central difference type finite-volume scheme. Also similarly to the fluid equation (Eq.4), the semi-discretised conduction equation for the solid temperature at stage $\mathrm{k}$ of the 4stage Runge-Kutta integration at time step m can be simply expressed as,

$$
\left(T_{s}\right)_{m}^{k}=\left(T_{s}\right)_{m-1}+c_{k} \Delta t_{S} R_{S}\left[\left(T_{s}\right)_{m}^{k-1}\right]
$$

The time step size $\Delta t_{S}$ useable for the solid domain needs to be emphasized. For a directly coupled unsteady CHT in time domain, the time steps for the fluid and solid domains needs to be the same, $\Delta t_{s}=\Delta t_{f}$. For a steady solid domain solution, the conduction equation is effectively marched in pseudo time, then $\Delta t_{S}$ is mesh-size and solid property dependent. It needs only to be restricted by the numerical stability (the Fourier number limit). For RANS solutions for turbine blade applications, an allowable time-step for solid conduction can be by up to 4 orders of magnitude larger than that for fluid convection (He and Oldfield, 2011). 


\section{Fluid-Solid Interface Treatment for CHT Solution}

The fundamental physical requirement for a fluid-solid interface is the heat flux and temperature continuity, Perelman (1961):

$$
\begin{gathered}
q_{f}=q_{s} \\
\left(T_{w}\right)_{f}=\left(T_{w}\right)_{s}=T_{w}
\end{gathered}
$$

We will first look at the baseline directly coupled interface treatment. Then the present moving-average based interface treatment will be introduced.

\subsection{Directly Coupled CHT Interface Treatment}

Take a fluid solid interface as shown in Fig.1, the baseline directly coupled interface condition requires that the physical flux and temperature continuity in the discretized forms is enforced at each and every iteration step (every stage of the 4-stage Runge-Kutta integration). For iteration step $k$, the one-sided finite difference representation of the heat fluxes on both sides for the heat flux continuity (7a) leads to an explicit expression of the wall temperature in terms of the fluid and solid temperatures at the centres of two adjacent mesh cells on the two sides,

$$
T_{W}^{k}=\frac{T_{f}^{k}\left(\frac{k_{f}}{\Delta x_{f}}\right)+T_{s}^{k}\left(\frac{k_{s}}{\Delta x_{s}}\right)}{\left(\frac{k_{f}}{\Delta x_{f}}\right)+\left(\frac{k_{s}}{\Delta x_{s}}\right)}
$$

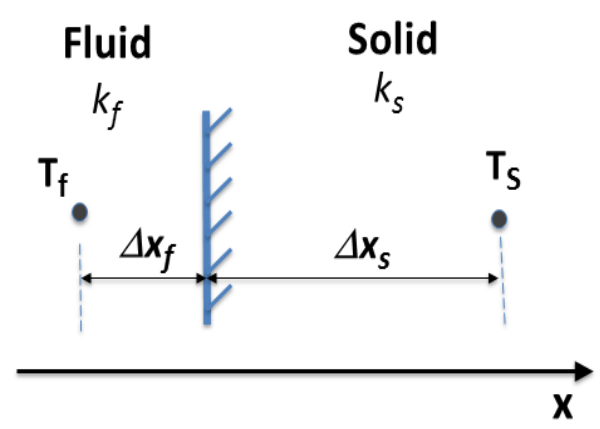

Fig.1, Interface between Fluid and Solid Domains

Define a 'mesh Biot number', $\mathrm{Bi}_{\mathrm{m}}$, as the local thermal resistance ratio between the solid mesh cell and the fluid one,

$$
B i_{m}=\left(\frac{\Delta x_{s}}{k_{s}}\right)\left(\frac{\Delta x_{f}}{k_{f}}\right)^{-1}
$$

Then we have

$$
T_{W}^{k}=\frac{B i_{m} T_{f}^{k}+T_{s}^{k}}{B i_{m}+1}
$$

The wall temperature obtained from Eq.10 is then used for both the fluid and solid domains simultaneously for marching forward to next iteration step $k+1$. Therefore the directly coupled treatment can be regarded as a Dirichlet-Dirichlet interface condition.

In the present work, the directly coupled interface method is used in two modes: 
i) $\quad$ Time-desynchronised Direct Coupling.

In this mode, the local time stepping is utilised, so that the CHT solution is time inconsistent $\left(\Delta \mathrm{t}_{\mathrm{f}} \neq \Delta \mathrm{t}_{\mathrm{s}}\right)$. This mode works very effectively for a pure steady CHT problem, where the time consistency and accuracy are not of concern. The timedesynchronised direct coupling is also very effective in propagating out and removing initial numerical transients. Thus, for the present LES based CHT solutions, the timedesynchronised direct coupling, denoted as 'Directly Coupled (Desynchronised)', will be used only to provide an initial field.

\section{ii) $\quad$ Time-synchronised (Time-consistent) Direct Coupling}

A time accurate CHT solution requires $\Delta \mathrm{t}_{\mathrm{f}}=\Delta \mathrm{t}_{\mathrm{s}}$. This is clearly problematic even just in terms of the computational costs given the huge time scale disparity. There is also the issue related to the extra mesh dependence when high frequency turbulence disturbances are resolved in LES, to be further illustrated in the present case studies. In the present work, the time-consistent directly coupled interface, denoted as 'Directly Coupled (Synchronised)', will be only used to provide baseline reference solutions for comparison purposes. The flow-chart for the directly coupled CHT method is shown in Fig.2.

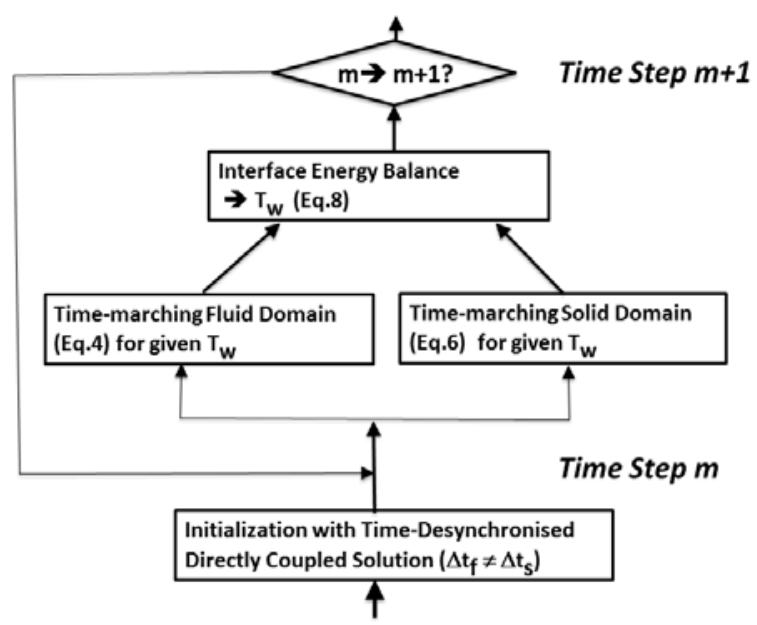

Fig.2, Process Flow Chart (Direct Coupled Interface)

\subsection{Fourier Spectral Interface Treatment}

The basic approach is motivated by that Fourier spectrum is commonly used for turbulence. Here a periodicity as implied for a Fourier representation is assumed to exist only for a very long time scale, much longer than those turbulence scales of interest. A general discrete Fourier spectrum with $\mathrm{N}_{\mathrm{F}}$ harmonics is in form of:

$$
U(x, t)=\widetilde{U}(x)+\operatorname{Real}\left(\sum_{n=1}^{N_{F}} \widehat{U}_{n} e^{i n \omega_{0} t}\right)
$$

where frequency $\omega_{0}$ is taken to set the longest time scale to be covered. $\widehat{U}_{n}$ is complex number coefficient for the nth harmonic. $\widetilde{U}(x)$ is the time-averaged part, the zeroth harmonic of the Fourier spectrum. 
The flow decomposition, expressed in the Fourier spectrum, provides a rational basis for treating the time-mean (steady) state and unsteady parts in different ways, although the two parts are dependent on each other. The approach taken here is to establish the time-averaged CHT interface first. This is the most important part of the interface treatment, because the timemean thermal characteristics are of primary practical interest. In addition, the time-averaged part also forms the sound basis on which the unsteady Fourier harmonics can be worked out. In the following sub-sections the time-averaged fluid-solid interface will be described first, followed by two important aspects of the unsteady part of the interface treatment, i) harmonic temperature-flux transfer function, and ii) Fourier transform of the fluid temperatures.

\subsubsection{Coupling Time-averaged Fluid Side with Solid Side (Zeroth Fourier Mode)}

By coupling the time-averaged fluid side with the solid side, the solid domain will completely avoid interacting with high frequency turbulence disturbances. As such the solid domain can be solved in the same way as for a steady CHT problem, with much larger local time steps limited only by the numerical stability requirement.

With time-average fluid parameters, the flux and temperature continuity should then read:

$$
\begin{aligned}
& \tilde{q}_{f}=q_{s} \\
& \left(\tilde{T}_{w}\right)_{f}=\left(T_{w}\right)_{s}=T_{w}
\end{aligned}
$$

A one-sided finite difference for the flux continuity (neglecting thermal radiation) is:

$$
\left(\frac{1}{\Delta x_{f}}\right)\left(\widetilde{k_{f} T_{f}}-\widetilde{k_{f}} T_{w}\right)=\left(\frac{k_{s}}{\Delta x_{s}}\right)\left(T_{w}-T_{s}\right)
$$

Thus:

$$
\widetilde{T}_{w}=\frac{\left(\frac{\widetilde{k_{f} T_{f}}}{\Delta x_{f}}+\frac{k_{s} T_{s}}{\Delta x_{s}}\right)}{\left(\frac{\widetilde{k_{f}}}{\Delta x_{f}}+\frac{k_{s}}{\Delta x_{s}}\right)}
$$

The wall temperature obtained from the time-averaged flux continuity (energy balance) and wall temperature continuity (Eq.12) is then used to time-march solutions of the mesh cells on both sides of the interface. Thus, this CHT coupling is also a 'Dirichlet-Dirichlet' type of interface treatment.

The time-averaging for the variables at the fluid mesh cell adjacent to the interface can be carried out in a 'moving-average', to denote that the average is effectively updated continuously at every time step. For a current time step N, the averaged fluid variable can be simply updated from the averaged one at the previous step and the current solution, e.g. He (2008):

$$
\widetilde{U}_{N}=\left(\frac{N-1}{N}\right) \widetilde{U}_{N}+\frac{1}{N} U_{N}
$$

As such, only would the time-averaged variables for the previous time step need to be stored, and the information required is completely local.

It should be noted that the time-averaging should be started after the initial transients otherwise convergence to the final time-averaged state would take much longer to reach. The convergence is effectively accelerated by running the time-desynchronised direct-coupling mode to provide an initial field first before starting the moving-averaging. The weighting between the previous average and the current solution in Eq.15 can also be changed by restarting with a different value of $\mathrm{N}$ to reduce the history 'memory' effect for a faster convergence. 


\subsubsection{Harmonic Transfer Function for Unsteady Wall Temperature}

When the unsteady flow disturbances are expressed in a discrete Fourier spectrum, the energy conservation can be achieved by balancing the time-average flux (as shown in Eqs.1214) and all unsteady harmonic components contained in the Fourier spectrum respectively. The corresponding flux continuity for the unsteady part can be expressed in harmonic balance (balancing each and every harmonic in complex number respectively):

$$
\left(\hat{q}_{f}\right)_{n}=\left(\hat{q}_{s}\right)_{n} \quad\left(\mathrm{n}=1,2, \ldots \ldots, \mathrm{N}_{\mathrm{F}}\right)
$$

Assuming a local 1D unsteady conduction behaviour for a semi-infinite solid domain, as in well-established date processing methods in transient heat transfer experiments, we can then make use of the analytical relation between the wall temperature and the heat flux for each and every harmonic on the solid wall:

$$
\left(\hat{q}_{s}\right)_{n}=\left(\hat{C}_{T q}\right)_{n}\left(\hat{T}_{s}\right)_{n} \quad\left(\mathrm{n}=1,2, \ldots \ldots, \mathrm{N}_{\mathrm{F}}\right) .
$$

where the complex number $\left(\hat{C}_{T q}\right)_{n}$ is regarded as a temperature-flux ('T-q') transfer function, entirely determined by solid material properties (assumed to be constant) and the frequency of harmonic $n$.

Eq.17 and the formulation for the T-q transfer function can be obtained by following the well-established procedure of Schultz and Jones (1973). It is noted however that the Schultz and Jones formulations are in a Laplace space, whilst the formulation for the present work needs to be for harmonics directly in frequency domain. Thus the difference between the present formulations and those of Schultz and Jones (1973) has to be clarified. In Appendix, the present procedure in terms of wall temperature and flux harmonics is presented. It also includes detailed formulations of the transfer function $\left(\hat{C}_{T q}\right)_{n}$ for solid domain with and without a thin layer TBC.

Given the analytical harmonic T-q transfer function (Eq.17) for the solid side (noting unsteady temperature continuity, $\left.\left(\widehat{T}_{s}\right)_{n}=\left(\widehat{T}_{w}\right)_{n}\right)$, we now only need a discrete (one sided finite difference) treatment for the fluid flux. The heat flux harmonic balance (Eq.16) then leads to a semi-analytical expression of the wall temperature harmonic:

$$
\left(\hat{T}_{w}\right)_{n}=\frac{k_{f}\left(\hat{T}_{f}\right)_{n}}{k_{f}+\Delta x_{f}\left(\hat{C}_{T q}\right)_{n}} \quad\left(\mathrm{n}=1,2, \ldots \ldots, \mathrm{N}_{\mathrm{F}}\right) .
$$

Assuming constant solid properties, the T-q transfer function $\left(\hat{C}_{T q}\right)_{n}$ can be worked out prior to a CHT solution. Then the only required input to Eq.18 for obtaining the wall temperature fluctuation harmonic is the fluid temperature harmonic $\left(\hat{T}_{f}\right)_{n}$ at the centre of fluid mesh cell adjacent to the interface (Fig.1). The fluid temperature harmonic will be obtained by the Fourier transform of the fluid temperature on the fly as described in the next sub-section.

The wall temperature harmonics (Eq.18), and the time-mean (zeroth harmonic) wall temperatures (Eq.14) are then used to construct the instantaneous unsteady wall temperature in time to proceed with the time-domain LES solution. As such, the Fourier spectral interface coupling effectively provides a Dirichlet condition in time domain at every time step for the fluid domain.

An interesting question to ask is, what happens to the unsteady conduction solution in the solid domain? It turns out to be perhaps the most remarkable feature of using the harmonic transfer function. We can now get unsteady wall temperatures without even solving the unsteady 
conduction equation in the solid domain at all. This is simply because the T-q transfer function (Eq.17) has analytically included the unsteady interaction between the heat fluxes on the wall and the temperatures in the solid domain, when combined with the discrete form of harmonic balance for the flux continuity.

Further comments are made on the 1-D semi-infinite solid conduction model as commonly adopted for gas turbine transient heat transfer experiments (Schultz and Jones 1973). For a temperature disturbance $\Delta \mathrm{T}_{\mathrm{f}}$ of frequency f due to large turbulence eddies, the spatial scales in a wall lateral direction should be comparable to boundary layer thickness. In the wall normal direction, however, a meaningful scale is the penetration depth, e.g. Faghri et al (2010):

$$
\delta_{p}=\sqrt{8 \alpha_{s} / f}
$$

For most conditions of practical interest, the penetration depth would be much smaller than a boundary layer thickness. Thus the 1D semi-infinite assumption is justifiable for large scale turbulence by a scaling analysis of the unsteady conduction equation in the wall normal and tangential directions.

For short scale turbulence disturbances, we may need to consider a discretised conduction equation for a near-wall solid mesh cell. The spatial variation of the disturbances in a wall tangential direction will be resolved by mesh resolution as expected. We are only concerned about the evaluation of the flux across the boundary face of the mesh cell on the solid wall surface. If the penetration depth is comparable to or smaller than the mesh spacing, the flux on the wall boundary face of the mesh cell can be quite accurately evaluated by neglecting the local variation in the wall tangential directions. As such, the 1-D semi-infinite model should be again deemed justifiable when used locally for the flux across a wall boundary face of a near wall solid mesh cell.

\subsubsection{Fourier Transform of Fluid Temperature}

To provide the input for working out unsteady wall temperature harmonic (Eq.18), we carry out a discrete Fourier transform of the fluid temperatures at the mesh cell adjacent to the interface (Fig.1). For a discrete Fourier transform, a real number expression is preferred for simplicity as previously applied to deterministic unsteady turbomachinery flows (He, 1992):

$$
T_{f}(x, t)=\tilde{T}_{f}(x)+\sum_{n=1}^{N_{F}}\left[A_{n} \cos \left(n \omega_{0} t\right)+B_{n} \sin \left(n \omega_{0} t\right)\right]
$$

with the complex-real number harmonics conversion, $\widehat{T}_{f n}=\left(T_{f n}\right)_{r}+i\left(T_{f n}\right)_{i}=A_{n}-i B_{n}$. As mentioned in the introduction, to completely avoid the aliasing errors, the Fourier transform of the fluid temperatures should be carried out by sampling at every time step. Setting the fluid time step $\Delta t_{f}$ and $\omega_{0}$ for the longest time scale to be covered, we get the number of time steps $\mathrm{N}_{0}$ to cover the time scale. For current time step $\mathrm{N}$, we also make use of the time-average temperatures obtained from the moving-average (Eq.15). Then the Fourier coefficients can be expressed in terms of the discrete Fourier transform of the temperatures for $\mathrm{N}_{0}$ steps prior to step $\mathrm{N}$ :

$$
\begin{aligned}
& \left(A_{n}\right)_{N}=\frac{\omega_{0} \Delta t_{f}}{\pi} \sum_{N-N_{0}}^{N}\left(T_{f}-\tilde{T}_{f}\right) \cos \left(n \omega_{0} t\right) \\
& \left(B_{n}\right)_{N}=\frac{\omega_{0} \Delta t_{f}}{\pi} \sum_{N-N_{0}}^{N}\left(T_{f}-\tilde{T}_{f}\right) \sin \left(n \omega_{0} t\right)
\end{aligned}
$$

To enable a continuous updating of the Fourier coefficients, we make use of existing Fourier coefficients to approximate the flow variables. This is the 'partial substitution' technique adopted in the early work by He (1992) to enable more frequent updating of the Fourier 
transform during a time marching solution. A continuous updating at every time step can be achieved by a reformulation of Eq.21 (e.g. shown by Gerolymos et al (2002) in a complex number form):

$$
\begin{aligned}
& \left(A_{n}\right)_{N}=\left(A_{n}\right)_{N-1}+\frac{\omega_{0} \Delta t_{f}}{\pi}\left[\left(T_{f}-\tilde{T}_{f}\right)_{N}-\left(T_{f}-\tilde{T}_{f}\right)_{N-N_{0}}\right] \cos \left(n \omega_{0} t\right) \\
& \left(B_{n}\right)_{N}=\left(B_{n}\right)_{N-1}+\frac{\omega_{0} \Delta t_{f}}{\pi}\left[\left(T_{f}-\tilde{T}_{f}\right)_{N}-\left(T_{f}-\tilde{T}_{f}\right)_{N-N_{0}}\right] \sin \left(n \omega_{0} t\right)
\end{aligned}
$$

Using the partial substitution technique, we approximate the unsteady temperatures at step $\mathrm{N}$ $\mathrm{N}_{0}$ by constructing the time-domain temperatures using the existing Fourier coefficients. As a result, the Fourier coefficients can be updated at every step (Eq.22) similarly to the time-mean counterpart (Eq.15), in a moving-average.

For the present implementation, once the lowest frequency $\omega_{0}$ for the longest time scale is chosen, the other frequencies are taken to be multiples of $\omega_{0}$. In terms of computational effort, typically if we retain 50-100 harmonics, about $30 \%$ extra computing time will be consumed with the current implementation.

Fig.3 shows the flow chart for the Fourier spectral interface coupling (denoted as 'Moving-average (Fourier-Spectral)'). The present methodology and implementation underscore the key features pursued. The most significant one is that the solid domain is solved essentially like a steady conduction problem with its local time steps dictated by numerical stability. As there is no unsteady conduction solution required in the solid domain, the associated mesh dependency discussed earlier is avoided. Also noted is that the harmonic transfer function makes use of the harmonic temperatures of the near wall fluid mesh cells generated by the Fourier transform on-the-fly sampled at every physical time step, to avoid the aliasing errors as intended.

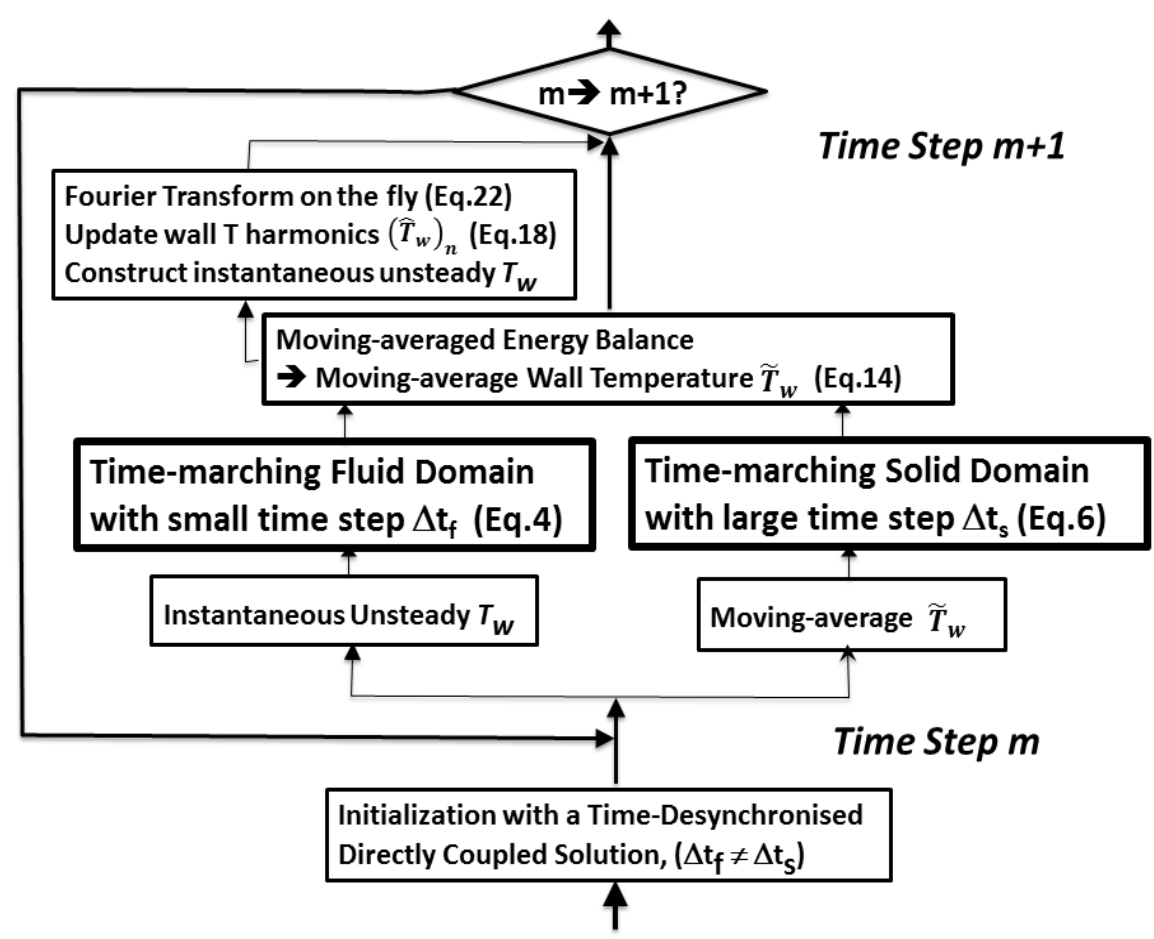

Fig.3, Process Flow Chart (Fourier Spectral Moving-average Interface Coupling 


\section{Stability of Moving-average based CHT Interface}

Next, we conduct some illustrative analyses on the stability behaviour of the proposed interface treatment. It is well known that a directly coupled CHT interface is stable as long as the stability requirements for both interior domains on the fluid side and the solid side are met, Giles (1997). The directly coupled interface method determines the wall temperature to enable the solutions on both sides to proceed, so it is effectively a Dirichlet-Dirichlet interface treatment. Here, we will employ a simple method for stability analysis and consider only the moving-average with the zeroth Fourier mode, as this is the only fluid part that the solid domain will interact with. Firstly we will establish the stability requirements for the fluid and solid interior domains respectively. In a similar way to previously established analyses, we will aim to show that for certain conditions considered in the present analysis for simplicity, the stability of a directly coupled CHT interface is assured by requiring no more stringent conditions than the interior domains. Then we will be able to show that a moving-average based CHT interface should work stably in a similar fashion for those conditions considered.

\subsection{Model Equations and Stability for Interior Domain}

Given the flow near a wall interface is dominated by diffusion in the wall normal direction, the fluid and solid governing equations can now be simplified to a 1-D energy equation with heat conduction terms only for the fluid side and a 1-D conduction equation for the solid side with constant fluid and solid properties, as in Giles (1997).

$$
\begin{aligned}
& \text { Fluid: } \frac{\partial T_{f}}{\partial t}=\alpha_{f} \frac{\partial^{2} T_{f}}{\partial x^{2}} \\
& \text { Solid: } \frac{\partial T_{s}}{\partial t}=\alpha_{s} \frac{\partial^{2} T_{s}}{\partial x^{2}}
\end{aligned}
$$

Now we take a forward Euler scheme for the temporal derivative and the central difference for the spatial one (with ' $\mathrm{j}$ ' denoting the mesh cell index). For either the fluid or the solid equation, we have for time step $\mathrm{N}$,

$$
\frac{T_{j}^{N+1}-T_{j}^{N}}{\Delta t}=\alpha \frac{T_{j+1}{ }^{N}-2 T_{j}^{N}+T_{j-1}{ }^{N}}{\Delta x^{2}}
$$

For the 1-D thermal diffusion/conduction, the steady temperatures vary linearly in either the fluid or the solid domain respectively. Now we introduce a saw-tooth type of error disturbances so that an instantaneous solution can be expressed as:

$$
T_{j}^{N}=T_{0 j}+\varepsilon_{j}{ }^{N}
$$

$\mathrm{T}_{0 \mathrm{j}}$ is the temperature of a stably converged discrete solution at mesh point $\mathrm{j}$. For the saw-tooth error distribution, we have

$$
\varepsilon_{j}{ }^{N}=-\varepsilon_{j+1}{ }^{N}=-\varepsilon_{j-1}{ }^{N},
$$

The temperatures $T_{0 j}$ of the converged solution will balance themselves on both sides of the discrete equations. The remaining terms form an equation for the error development.

$$
\varepsilon_{j}{ }^{N+1}=\varepsilon_{j}{ }^{N}+\frac{\alpha \Delta t}{\Delta x^{2}}\left(\varepsilon_{j+1}{ }^{N}-2 \varepsilon_{j}{ }^{N}+\varepsilon_{j-1}{ }^{N}\right)
$$


Making use the saw-tooth error relation (Eq.26) and noting that errors need to decay in time, $\left|\frac{\varepsilon_{i}^{N+1}}{\varepsilon_{i}}\right|<1$ for stability, we thus have for either the fluid domain or the solid domain:

$$
0<\frac{\alpha \Delta t}{\Delta x^{2}}<\frac{1}{2}
$$

\subsection{Stability of Directly Coupled Fluid-Solid Interface}

\subsubsection{Interface Error Equation}

We now make use of the simple perturbation analysis for a directly coupled interface, to verify its usefulness in identifying the conditions for the stability as known, Giles (1997). Express the temperatures at the two sides of the interface as those of the exact solution and errors respectively:

$$
\begin{aligned}
& T_{f}{ }^{N}=T_{f 0}+\varepsilon_{f}{ }^{N} \\
& T_{w}{ }^{N}=T_{w 0}+\varepsilon_{w}{ }^{N} \\
& T_{s}{ }^{N}=T_{s 0}+\varepsilon_{s}{ }^{N}
\end{aligned}
$$

The discrete form of the flux continuity across the interface, after balancing the terms of the exact solution, becomes:

$$
k_{f} \frac{\varepsilon_{f}^{N}-\varepsilon_{w}^{N}}{\Delta x_{f}}=k_{s} \frac{\varepsilon_{w}^{N}-\varepsilon_{s}^{N}}{\Delta x_{s}}
$$

Then the wall temperature error can be expressed in terms of those at the fluid and solid mesh points and the mesh Biot number, $B i_{m}$ (Eq.10):

$$
\varepsilon_{w}^{N}=\frac{B i_{m} \varepsilon_{f}^{N}+\varepsilon_{s}^{N}}{B i_{m}+1}
$$

We now consider two extreme $\mathrm{Bi}_{\mathrm{m}}$ cases to illustrate the stability condition.

\subsubsection{Stability of Directly Coupled Interface for $B i_{m}=0$.}

From the wall error equation (Eq.30), we then have, $\varepsilon_{w}^{N}=\varepsilon_{s}^{N}$.

Take $\mathrm{j}=0$ at the wall, $\mathrm{j}>0$ for the solid wide and $\mathrm{j}<0$ for the fluid side, as shown in Fig. 4 . Then the error equation (Eq.27) for the adjacent mesh cell on the solid side becomes:

$$
\varepsilon_{1}{ }^{N+1}=\varepsilon_{1}{ }^{N}+\alpha_{s} \frac{\Delta t_{s}}{\Delta x_{S}^{2}}\left(\varepsilon_{2}{ }^{N}-2 \varepsilon_{1}{ }^{N}+\varepsilon_{w}{ }^{N}\right)
$$

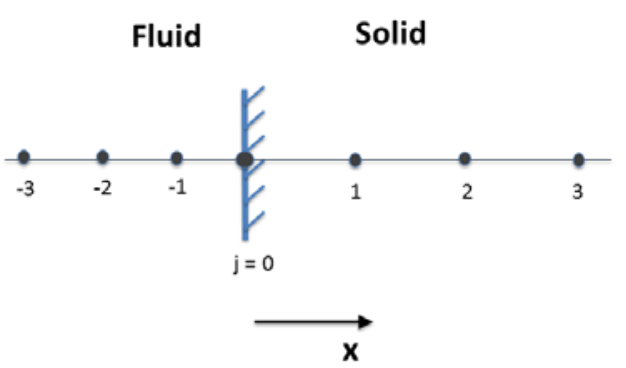

Fig.4, Mesh Cell Indexing around Fluid-Solid Interface 
Given the saw-tooth error distribution, we have, $\varepsilon_{2}{ }^{N}=-\varepsilon_{1}{ }^{N}=-\varepsilon_{w}{ }^{N}$.

For stability: $\left|\frac{\varepsilon_{1}{ }^{N+1}}{\varepsilon_{1}{ }^{N}}\right|<1$, which is equivalent to : $\left|\frac{\varepsilon_{w}^{N+1}}{\varepsilon_{w}{ }^{N}}\right|<1$,

The stability condition required thus is:

$$
0<\frac{\alpha_{s} \Delta t_{s}}{\Delta x_{s}^{2}}<1
$$

This is clearly a less stringent condition than that required for the stability of the interior solid domain (Eq.28). Thus at this condition $\left(B i_{m} \approx 0\right)$, a stability condition for the solid domain solution should be sufficient for a stable directly coupled interface treatment.

\subsubsection{Stability of Directly Coupled Interface for $B i_{m}=\infty$,}

From the wall error equation (Eq.30), we now have, $\varepsilon_{w}^{N}=\varepsilon_{f}^{N}$. Then the error equation (Eq.27) for the adjacent mesh cell in the fluid domain becomes (given the mesh indices as shown in Fig.4):

$$
\varepsilon_{-1}{ }^{N+1}=\varepsilon_{-1}{ }^{N}+\alpha_{f} \frac{\Delta t_{f}}{\Delta x_{f}^{2}}\left(\varepsilon_{w}{ }^{N}-2 \varepsilon_{-1}{ }^{N}+\varepsilon_{-2}{ }^{N}\right)
$$

In this case, we have $\varepsilon_{-2}{ }^{N}=-\varepsilon_{-1}{ }^{N}=-\varepsilon_{w}{ }^{N}$. Then similarly to the solid domain, we look at the stability for the fluid mesh point, $\mathrm{j}=-1$. The corresponding condition required for the decay of wall temperature error, $\left|\frac{\varepsilon_{w}^{N+1}}{\varepsilon_{w}^{N}}\right|<1$, is:

$$
0<\frac{\alpha_{f} \Delta t_{f}}{\Delta x_{f}^{2}}<1
$$

Eq.32 is also less stringent than that for the fluid interior domain (Eq.28). Thus, in this case, a stable condition of the interior fluid domain solution is sufficient for a stable interface.

Overall, we can see that the directly coupled interface will be stable as long as both fluid and solid interior domain solutions are stable. Note that a coupled CHT system is solved by a time-marching method, regardless if the problem is unsteady or not. The directly coupled interface works quite well for a steady CHT problem. As pointed out by He and Oldfield (2011), for explicit time marching CHT solutions, the disparity in the time step sizes as required by the numerical stability is compatible to that of the physical time scales between the two domains. Thus, without the time-consistency constraint, the numbers of time steps needed to propagate the physical disturbances in the two domains respectively can be compatible. Therefore a directly coupled time-marching CHT method is effective for a steady CHT solution. This is also a primary motivation to solve the solid domain in a steady manner.

\subsection{Stability of Moving-average based Fluid-Solid Interface}

In a similar way to that for the directly coupled interface, we aim to show that the stability of a moving-average interface would require no more stringent conditions than those required for stable interior domain solutions. The formulation and equation should be the same as those for a directly coupled interface except that the time-averaged fluid temperatures now are taken. For time step $\mathrm{N}$, the time-averaged temperature at a mesh point is taken from all fluid temperatures of the same mesh point at the previous time steps up to N. It can be also expressed in terms of the exact solution and a time-averaged error for this mesh point: 


$$
\widetilde{T}_{f}^{N}=T_{f 0}+\tilde{\varepsilon}_{f}^{N}
$$

The corresponding wall temperature error can then be expressed as:

$$
\varepsilon_{w}^{N}=\frac{B i_{m} \tilde{\varepsilon}_{f}^{N}+\varepsilon_{s}^{N}}{B i_{m}+1}
$$

As for the directly coupled interface treatment, we also consider the two $\mathrm{Bi}_{\mathrm{m}}$ conditions.

\subsection{1, Stability of Moving-average Interface for $B i_{m}=0$.}

We have, $\varepsilon_{w}^{N}=\varepsilon_{s}^{N}$. Note that the solid side for the moving-average interface treatment is the same as that for the directly coupled one. We can then follow the same procedure, reaching the same outcome. For stability, $\left|\frac{\varepsilon_{w}^{N+1}}{\varepsilon_{w}^{N}}\right|<1$, the condition required is:

$$
0<\frac{\alpha_{s} \Delta t_{s}}{\Delta x_{s}^{2}}<1
$$

So the interface stability at this condition is sufficiently assured when the numerical stability condition of the solid domain is satisfied.

\subsection{2, Stability of Moving-average Interface for $B i_{m}=\infty$.}

We now have, $\varepsilon_{w}^{N}=\tilde{\varepsilon}_{f}^{N}$. Thus the moving averaged interface treatment will be stable if the time-averaged errors on the fluid side $\tilde{\varepsilon}_{f}^{N}$ decay in time. For the saw-tooth error distribution in the fluid interior domain and given the mesh index convention adopted $(\mathrm{j}<0$ for the fluid domain, Fig.4), we then have, $\varepsilon_{f}{ }^{N}=\varepsilon_{-1}{ }^{N}=-\varepsilon_{-2}{ }^{N}$.

The error equation for the fluid mesh cell adjacent to the interface becomes:

$$
\varepsilon_{-1}{ }^{N+1}=\varepsilon_{-1}{ }^{N}+\alpha_{f} \frac{\Delta t_{f}}{\Delta x_{f}^{2}}\left(\varepsilon_{w}{ }^{N}-3 \varepsilon_{-1}{ }^{N}\right)
$$

Summing the equations for all previous time steps up to step N, we have:

$$
\tilde{\varepsilon}_{-1}{ }^{N+1}=\tilde{\varepsilon}_{-1}{ }^{N}+\alpha_{f} \frac{\Delta t_{f}}{\Delta x_{f}^{2}}\left(\tilde{\varepsilon}_{w}{ }^{N}-3 \tilde{\varepsilon}_{-1}{ }^{N}\right)
$$

In terms of the wall temperature errors, the above can be rewritten as:

$$
\varepsilon_{w}{ }^{N+1}=\varepsilon_{w}{ }^{N}+\alpha_{f} \frac{\Delta t_{f}}{\Delta x_{f}^{2}}\left(\tilde{\varepsilon}_{w}{ }^{N}-3 \varepsilon_{w}{ }^{N}\right)
$$

Now we need to relate the averaged wall temperature error to the current error at $\mathrm{N}$.

For stability, the requirement, $\left|\frac{\varepsilon_{w}^{m+1}}{\varepsilon_{w}^{m}}\right|<1(\mathrm{~m}=1,2, \ldots \mathrm{N})$, should hold for all steps prior to step $\mathrm{N}$. Also assume that the errors at the same mesh point have the same sign: $\left|\tilde{\varepsilon}_{w}{ }^{N}\right|=$ $\frac{1}{N} \sum_{m=1}^{N}\left|\varepsilon_{w}{ }^{m}\right|$. Then, the decaying magnitude of the wall errors with time step means, $\left|\tilde{\varepsilon}_{w}{ }^{N}\right|>$ $\left|\varepsilon_{w}{ }^{N}\right|$.We can thus express the time-averaged error in terms of the current error at step $\mathrm{N}$ :

$$
\tilde{\varepsilon}_{w}{ }^{N}=c_{N} \varepsilon_{w}{ }^{N},\left(c_{N}>1\right)
$$


The error growth ratio now becomes

$$
\varepsilon_{w}{ }^{N+1} / \varepsilon_{w}{ }^{N}=\left[1+\alpha_{f} \frac{\Delta t_{f}}{\Delta x_{f}^{2}}\left(c_{N}-3\right)\right]
$$

For the wall temperature errors to decay, we then have:
a) $\frac{\alpha_{s} \Delta t_{s}}{\Delta x_{s}^{2}}\left(3-c_{N}\right)>0$
b) $\frac{\alpha_{s} \Delta t_{s}}{\Delta x_{s}^{2}}\left(3-c_{N}\right)<2$

It can be shown, $1<\mathrm{c}_{\mathrm{N}}<3$. Consequently the corresponding stable condition is,

$$
0<\frac{\alpha_{s} \Delta t_{s}}{\Delta x_{s}^{2}}<\frac{2}{\left(3-c_{N}\right)} \quad, \quad\left(1<c_{N}<3\right)
$$

The condition given by Eq.34 is again less stringent than the stability condition for a stable solution of the interior fluid domain (Eq.28). We can therefore summarize that for the conditions considered here, the moving-average based Dirichlet-Dirichlet interface treatment should be stable if the stability conditions for the interior fluid and solid domains are satisfied.

\section{Impact of Interface Treatment on Wall Temperature and Heat Flux}

The interface methods presented in Section 3 have been implemented and tested for various cases. All numerical cases tested have been stable, consistent with the findings of the simple stability analysis. We will now examine and illustrate the impact of the interface treatment on solution consistency and accuracy.

The turbine blading configuration of NASA C3X, Hylton et al (1983) is taken here. It is a transonic turbine cascade with experimental measurements of surface pressure and heat transfer distributions. In the following, a validation of the unsteady flow solver is first presented. Then computational studies for conjugate heat transfer with the interface treatments will be presented and the unsteady wall shear stresses, wall temperatures and heat fluxes will be further examined.

\subsection{Validation of Flow Solver}

We consider a case at an exit Mach number of 0.9 and Reynolds number (based in inlet velocity) of $4 \times 10^{5}$. The original cascade blading is of $2 \mathrm{D}$ profile with a span/axial chord ratio of 1.1. The present calculations are conducted in a single blade passage domain for 3D blade sector with $10 \%$ span length. The direct periodic condition is applied at both the blade-to-blade periodic boundaries, and at the two spanwise boundaries.

The flow is subject to a clearly identifiable laminar to turbulent transition between $40 \%$ and $60 \%$ chordwise positions. The pressure surface is also subject to a transitional flow, although there seems no clearly identifiable location for the onset (Hylton et al, 1983). The computations are carried out by large eddy simulations. In total there are 3.4 million mesh cells for this $10 \%$ span sector, corresponding to $\sim 30$ million mesh cells for a blade passage. This mesh density can be regarded as being relatively coarse for LES of the given Reynolds number. The nonslip wall conditions is applied with the first mesh cells being within the laminar sub-layer with $\mathrm{y}^{+}$smaller than 5. The transitional flow characteristics are dealt with by tuning the coefficient of the eddy viscosity of the sub-grid model. For the clearly laminar flow, the coefficient is specified to be zero. In other zones where the boundary layer is deemed to be turbulent, the eddy viscosity coefficient is taken to vary within the normal range. 
It is noted that this case is subject to a $6 \%$ free stream turbulence. To accommodate this in the LES computation, synthetically generated velocity fluctuations are introduced at the inlet, as in the author's previous work (He, 2013). At the inflow boundary, the Fourier spectrum is adopted for generating synthetic turbulent velocity fluctuations in time domain. Each harmonic represents a simplified local vortical flow structure with its spatial length scale directly proportional to its temporal one (frequency). The phase angles of these locally regular vortical structures are randomized so that the inflow boundary appears to be injecting turbulence eddies in a random fashion. The shape of the harmonic spectrum is approximated by blinding an integral scale part of a constant amplitude and an initial scale region with a $-5 / 3$ slope.

Fig.5 presents the surface pressure distribution along the axial chord. The computational results are shown to agree well with the corresponding experimental data. For validating the convective heat transfer prediction capability of the flow solver without the complications of the conjugate heat transfer involved in the solid part, the heat transfer computation for this case is carried out with specified wall temperatures from the experiment (Hylton et al, 1983).

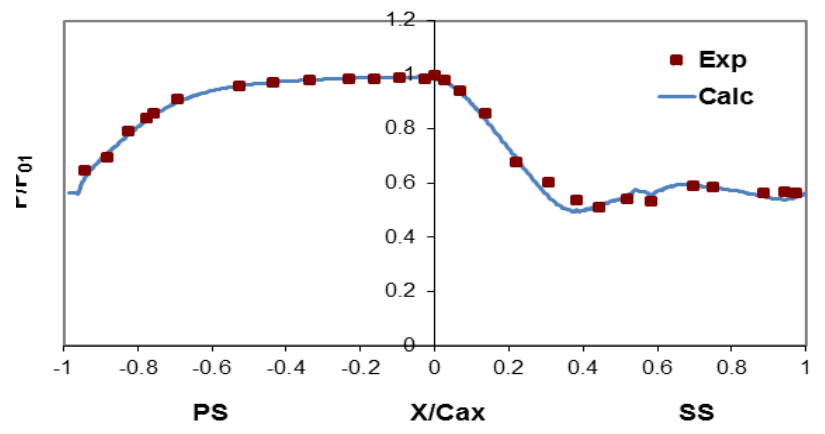

Fig.5, Surface Pressure Distributions for NASA C3X Turbine Cascade (normalised by inlet total pressure)

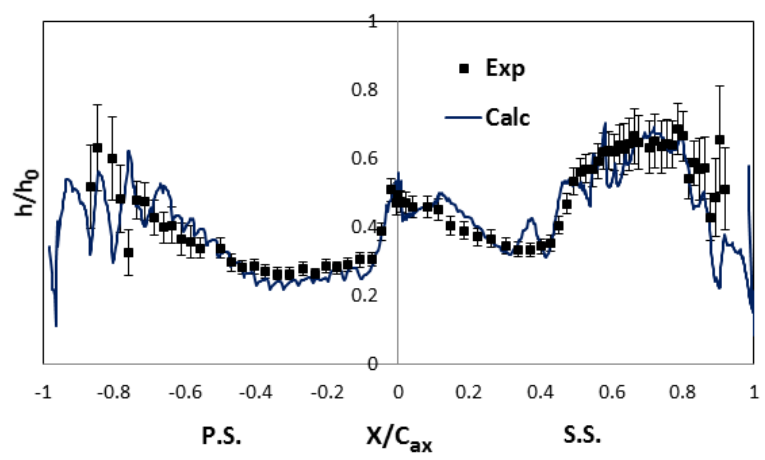

Fig.6, Surface Heat Transfer Coefficient Distributions for NASA C3X Turbine Cascade ( $\mathrm{h}_{0}=1135$, experimental uncertainties indicated by error bars)

Fig.6 shows the non-dimensional heat fluxes (in terms of the normalised HTC) between the computational and the experimental results. The present computed surface heat transfer distributions are deemed as satisfactory, particularly considering the uncertainty range of the experimental data (indicated by the error bars in Fig.6). The aerodynamic and heat transfer results of this validation case lead to sufficient confidence in the unsteady flow solver before the following computational studies on the impact of the CHT interface treatment are conducted. 


\subsection{Impact of CHT Interface Treatment}

First we examine the influence of the conjugate heat transfer. The fluid part of the configuration and the mesh are the same as one used in the previous test case for the LES flow solution with the specified wall temperatures. To test the CHT part, a solid domain will be needed. The CHT configuration is taken to approximate a simple internal cooled NGV blade with two main internal cooling channels. The configuration and the mesh are shown in Fig.7. The internal wall boundary condition is given as a convective one with a HTC of $500 \mathrm{~W} / \mathrm{m}^{2} \mathrm{~K}$. The inlet hot gas temperature is $800^{\circ} \mathrm{K}$, and the coolant temperature is taken to be $400^{\circ} \mathrm{K}$, with a typical hot gas/coolant temperature ratio of 2 of practical interest. The solid material is taken to be Nickel steel, with a density of $8000 \mathrm{~kg} / \mathrm{m}^{3}$, a thermal conductivity of $80 \mathrm{~W} / \mathrm{mK}$, and a specific heat of $450 \mathrm{~J} / \mathrm{kgK}$. The fluid time step for LES is around $0.02 \mu \mathrm{s}$. The through-flow time period for the blade domain takes about 10000 time steps. LES-CHT solutions typically are run for at least 20 through-flow time periods.

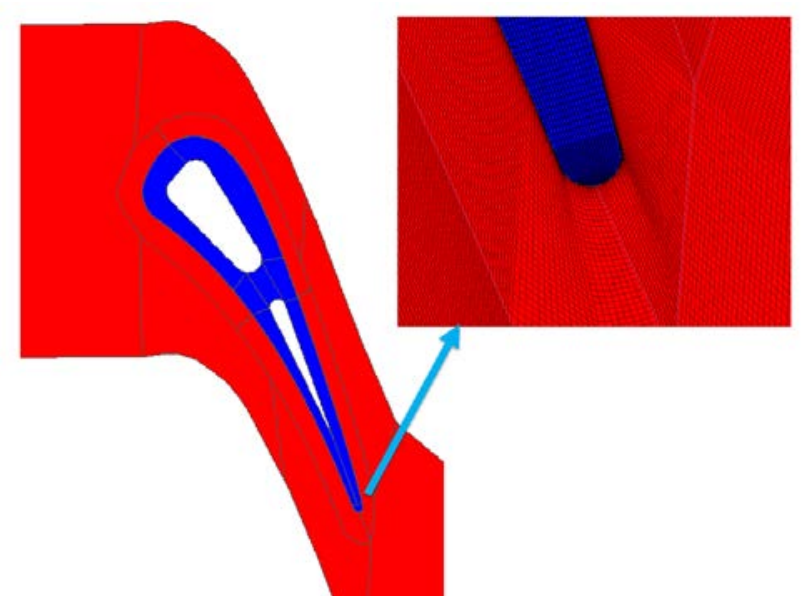

Fig.7, Conjugate Heat Transfer Configuration and Mesh

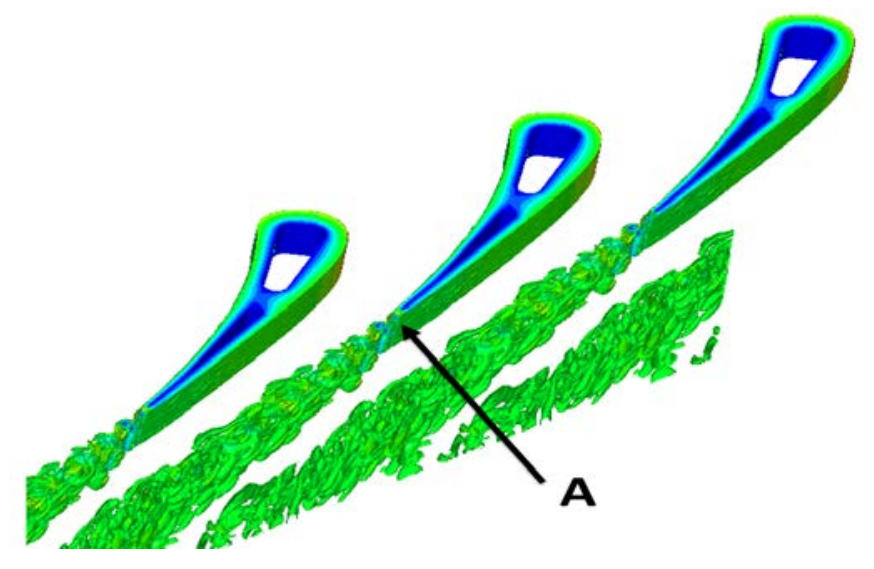

Fig.8, Unsteady Vortical Flow Pattern of Conjugate Heat Transfer Solution

It should be noted that although the mean inlet and exit flow conditions are the same as those taken in the previous test case, all the CHT computational studies are carried out with zero inflow turbulence. This inflow condition is taken to ensure that the unsteady flow fluctuations experienced in the interface are completely self-generated within a turbulent boundary layer, rather than due to the upstream synthetically generated unsteadiness. 
The resolving capability of the present LES for the turbulence flow is indicated by the instantaneous vortical flow patterns as shown in Fig.8. The location of particular interest is around the blade trailing edge. The impact of the wall heat transfer on the local boundary layer is expected to be the strongest, chiefly due to the 'history effect' of the accumulated wall cooling on the upstream boundary layer, as observed by Maffulli and He (2014). In the following we will examine some key aerothermal parameters (wall temperature and heat flux) as well as aerodynamic parameters (wall shear stress) at a near trailing edge location on the suction side (point A shown in Fig.8). This is where the flow is subject to both broad-band turbulence and coherent trailing edge vortex-shedding. We will look at some sample results in relation to the two aspects of the primary interest: a) the impact of the wall heat transfer on near-wall aerodynamic behaviour, and b) the impact of the interface treatment on accuracy and consistence of a turbulence scale resolved solution such as LES.

\subsubsection{Adiabatic vs. CHT Solutions}

First, we get some indication of the heat transfer impact on the aerodynamics, in particular in a relatively high heat flux condition at a realistic gas-wall temperature ratio typical of that for a high pressure gas turbine. It is of interest to look at the influence of wall heat transfer on the near-wall turbulence flow behaviour.

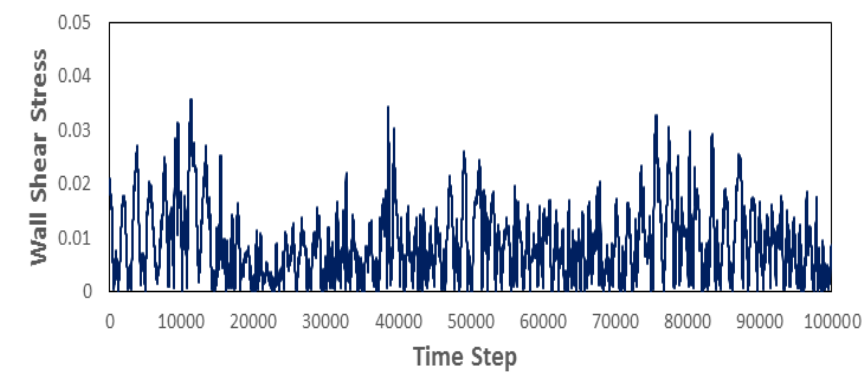

a) Adiabatic Wall Condition

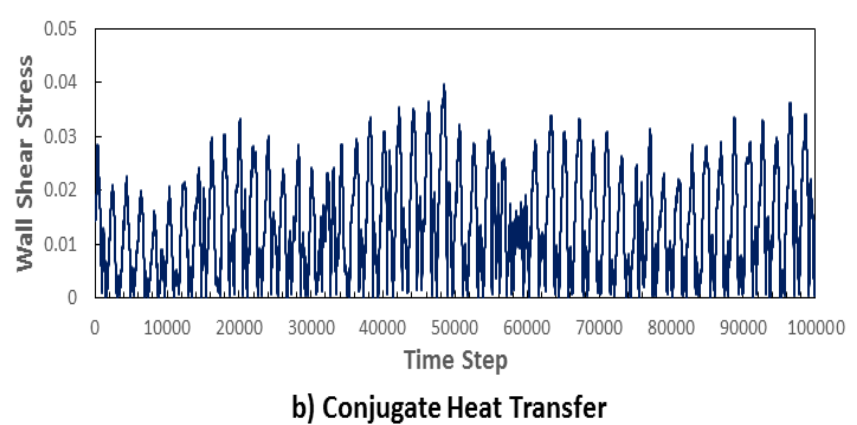

Fig.9, Time Traces of Wall Shear Stress at a Trailing-Edge point (Adiabatic vs. CHT).

The time traces of the wall shear stress (normalised by the inlet dynamic head) at point A (Fig.8) are shown in Fig.9. Here the time trace calculated by the CHT solution is compared to that with an adiabatic wall condition. There are clear differences between the two solutions. Firstly, unsteady characteristics of the CHT solution (Fig.9b) appear to be more coherent than that of the adiabatic solution (Fig.9a). Secondly, the time mean value of the shear stress for the CHT solution is markedly different (by about 50\% higher than that of the adiabatic solution). This behaviour is qualitatively in line with the observation from a previous steady RANS study 
by Maffulli and He (2014), showing a thinner boundary layer of a cooled wall compared to its adiabatic counterpart, with a maximum accumulated ('history') effect at the blade trailing edge. A thinner boundary layer of the cooled wall has equivalently a higher local Reynolds number in the near-wall region, thus higher local turbulence fluctuations of the CHT solution might be expected. In addition, a thinner boundary layer approaching the blade trailing edge of a finite thickness may promote a more vigorous vortex shedding, leading to more coherent unsteadiness than its adiabatic counterpart as observed here.

\subsubsection{Directly Coupled vs. Moving-Average Interface}

Next, we turn the attention to the impact of the interface treatment which is the main focus of the present work. CHT computations for the turbine blade case are carried out and the results are contrasted between the time-synchronised directly coupled interface and the Fourier spectral moving-average one. For the Fourier-spectral moving-average interface, 100 harmonics are taken with frequencies ranging from $2000 \mathrm{~Hz}$ to $200000 \mathrm{~Hz}$ at a constant interval of $2000 \mathrm{~Hz}$. Two cases are considered. The first one is a single metal layer of Nickel Steel. In the second case, the wall surface is subject to a thin layer of TBC.

\section{Single Solid Layer}

For a single metal solid domain without TBC, a typical mesh Biot number is about 0.01. The time traces of the wall temperatures at point A are shown in Fig.10. The corresponding time traces of the heat fluxes (normalised by the inlet fluid thermal conductivity, the blade trailing edge diameter and the inlet to local time-mean temperature difference) are shown in Fig.11.

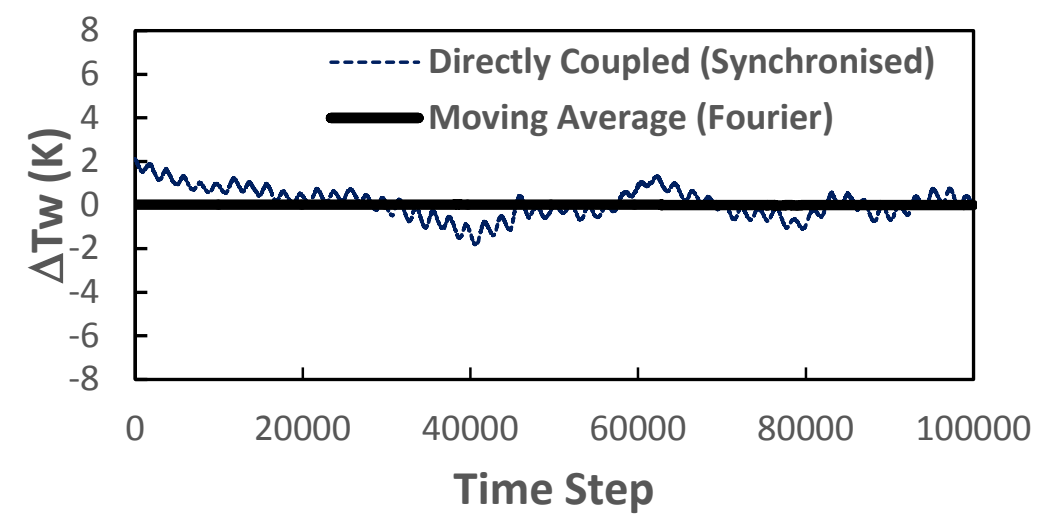

Fig.10, Time Traces of Wall Temperatures at a Trailing-Edge point (no TBC) (Synchronised Direct Coupling vs. Fourier Spectral Moving-average)

In the single layer case, the heat fluxes calculated by both interface methods are of a similar magnitude, though with different details. However, the time-synchronised directly coupled interface produces seemingly much higher wall temperature fluctuations than the movingaverage based (Fig.10). The wall temperature fluctuations predicted by the moving-average are all negligibly small $(<0.1 \mathrm{~K})$. For this case, the Fourier spectral model with only the simple time average (i.e. the zeroth harmonic only) produces almost the same results as that with 100 harmonics, given the insignificant wall temperature unsteadiness. 


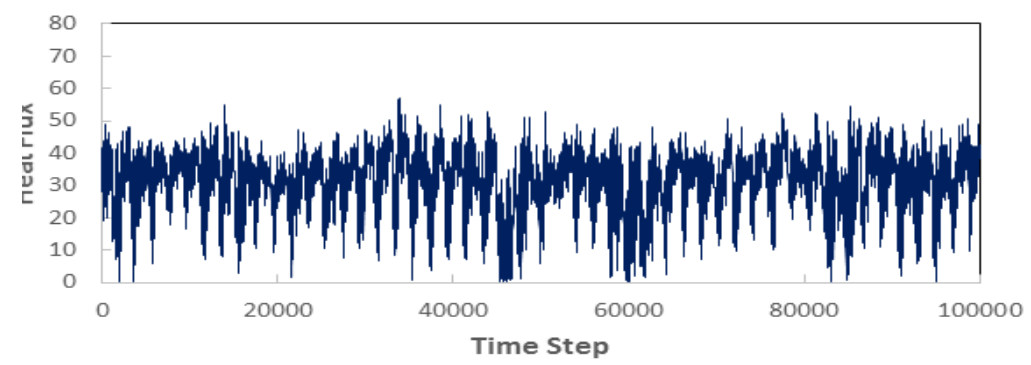

a) Directly Coupled (Synchronised)

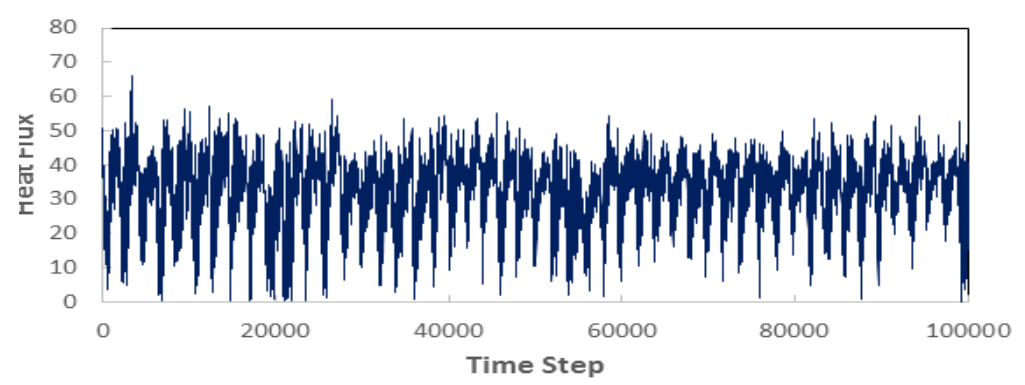

b) Moving Average (Fourier-Spectral)

Fig.11, Time Traces of Wall Heat Fluxes s at a Trailing-Edge point (no TBC) (Synchronised Direct Coupling vs. Fourier Spectral Moving-average).

Solid Layer with Thermal Barrier Coating (TBC)

We now have a TBC layer added to the solid surface. The thickness of the TBC is typically very thin, and is taken to be $0.1 \mathrm{~mm}$ in this case. The TBC material is taken to be a low conductivity Kapton with a density of $1420 \mathrm{~kg} / \mathrm{m}^{3}$, a thermal conductivity of $0.155 \mathrm{~W} / \mathrm{mK}$, and a specific heat of $1090 \mathrm{~J} / \mathrm{kgK}$. For this TBC case, a typical mesh Biot number is about 4.

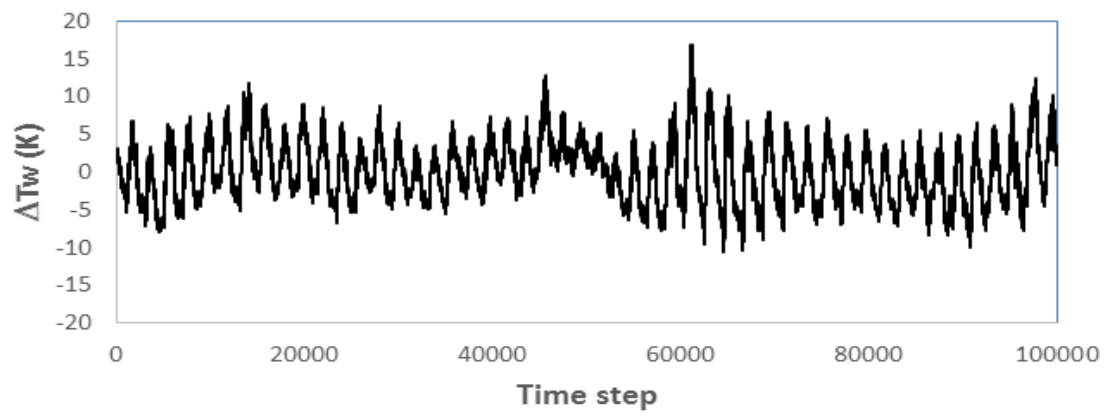

a) Directly Coupled (Synchronised)

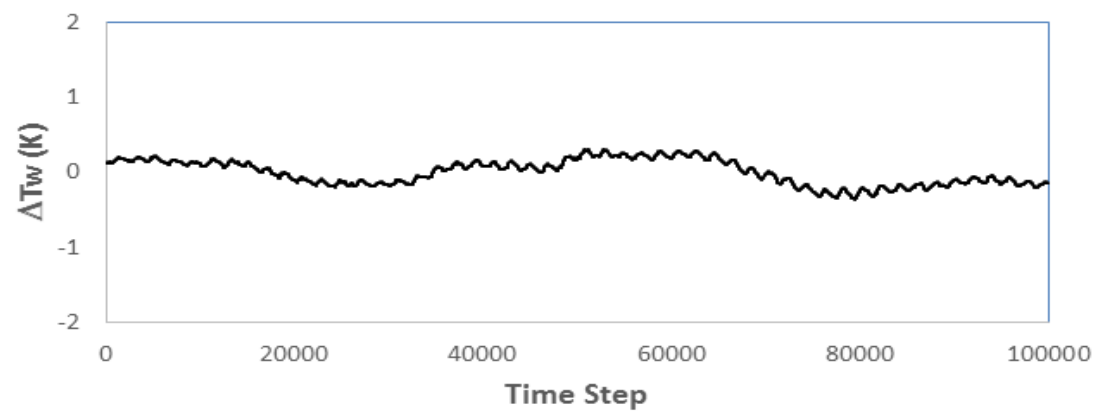

b) Moving Average (Fourier-Spectral)

Fig.12, Time Traces of Wall Temperatures at a Trailing-Edge point (with TBC), (Time-synchronised Direct Coupling vs. Fourier Spectral Moving-average). 
The calculated time traces of the wall temperatures at point $A$ are shown in Fig.12. The corresponding time traces of the non-dimensional heat fluxes are shown in Fig.13. With the TBC, we now see markedly different unsteady wall characteristics (Figs.12, 13) compared to those of the single layer solid without TBC (Figs.10, 11). The results of the time synchronised directly coupled interface solution show very large high frequency wall temperature fluctuations ( 10K, Fig.12a), one order of magnitude larger than those produced by the Fourier spectral interface based on the moving-average $(<0.5 \mathrm{~K}$, Fig. $12 \mathrm{~b})$. There is also a marked impact on the heat flux results. The directly coupled interface method produces much smaller wall heat flux fluctuations (Fig.13a), by a factor of 3 - 4, than those produced by the Fourier spectral movingaverage interface method (Fig.13b).

To get some insight into the over-prediction of wall temperature fluctuations by the directly coupled interface method, an interface response analysis is carried out. The analysis is similar to that adopted for a single solid domain subject to natural convection (Fadl and He, 2017). In the present work, the analysis is extended to a two-layer solid domain with TBC.

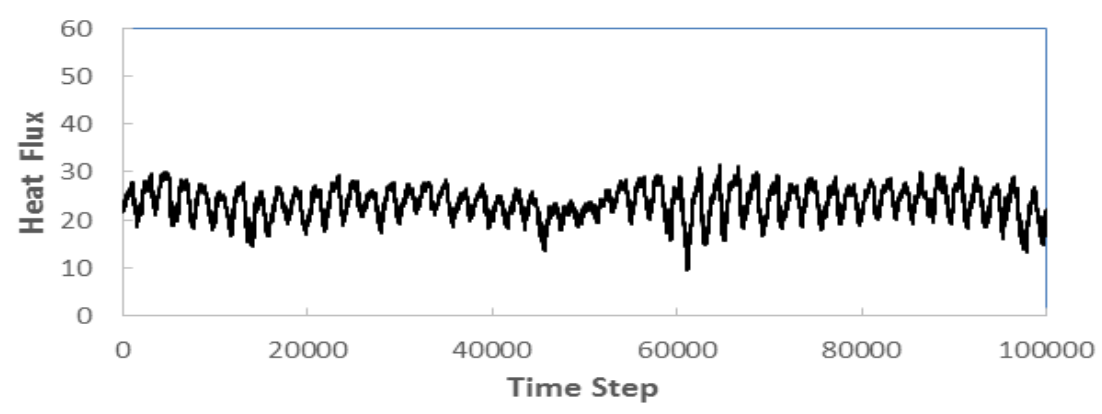

a) Directly Coupled (Synchronised)

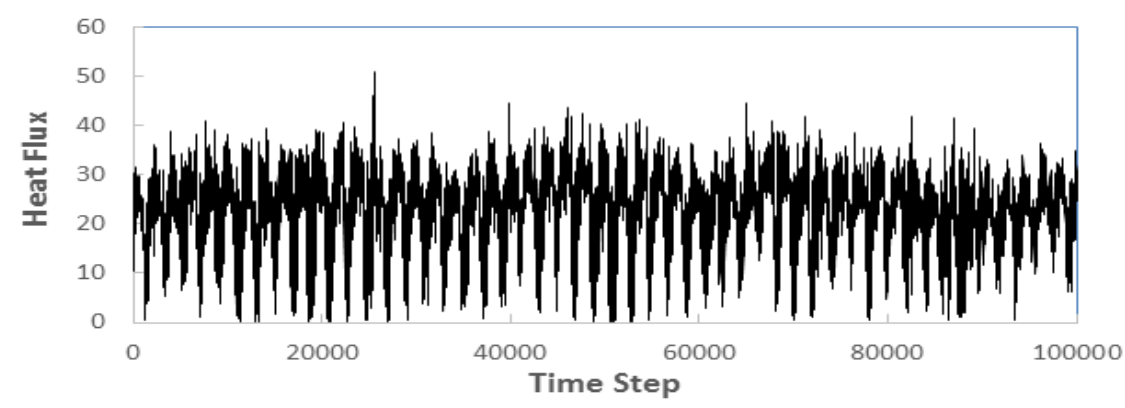

b) Moving Average (Fourier-Spectral)

Fig.13, Time Traces of Wall Heat Fluxes s at a Trailing-Edge point (with TBC)

(Time-synchronised Direct Coupling vs. Fourier Spectral Moving-average).

It is noted that the wall temperature formulation in terms of the fluid and solid temperatures (Eq.8) is a discrete form of the temperature and heat flux continuity at an interface. The finitedifference approximation of the heat flux balance between the two sides of the interface (Eq.7a) can only be deemed adequate if the penetration can reach the centre of the solid mesh cell adjacent to the interface. The interface response analysis examines the calculated (erroneous) temperature responses to the correct (target) values, for a given solid mesh. 
For a given unsteady fluid temperature disturbance $\Delta \mathrm{T}_{\mathrm{f}}$., we look at the wall temperature response, as shown in Fig.14. The TBC thickness $\Delta \mathrm{x}_{\mathrm{TBC}}$ is within one solid mesh spacing. It is thus accounted for by the corresponding thermal resistance of the two-layer composite solid mesh cell. For the given frequency range of interest, the penetration depth $\delta_{\mathrm{p}}$ of the temperature disturbances is mostly within the TBC layer $\left(\delta_{\mathrm{p}}<\Delta \mathrm{x}_{\mathrm{TBC}}\right)$.

The correct target wall temperature response should be $\Delta \mathrm{T}_{\mathrm{w}}$. However, in this case, the disturbance can only reach the distance marked by the penetration depth $\delta$ p. The solid mesh point thus cannot 'see' the disturbance. The discrete form of the wall temperature expression assumes that the disturbance should at least reach the solid mesh point (marked by the dash line in Fig.14). As a result, the unsteady wall temperature response $\Delta \mathrm{T}_{\mathrm{w}}{ }^{\prime}$ will be over-predicted, and the corresponding wall heat flux response (slope of the dashed line) will be under-predicted.

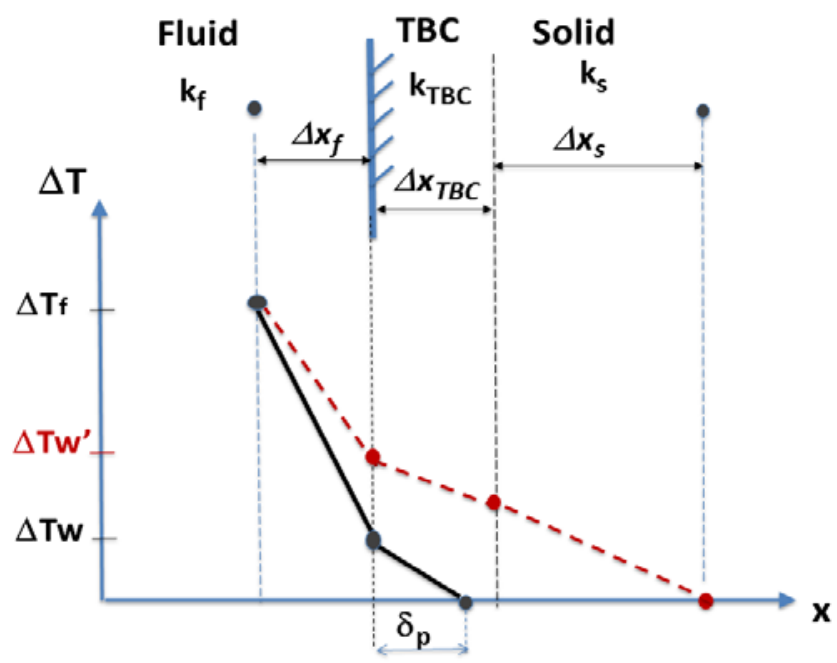

Fig.14, Wall Temperature Response to Fluid Temperature Disturbance $\Delta \mathrm{T}_{\mathrm{f}}$ (with TBC)

Use the discrete formulation to calculate the wall temperature (Eq.10). Balancing the steady base part, the calculated temperature response $\Delta \mathrm{T}_{\mathrm{w}}{ }^{\prime}$ (noting $\Delta \mathrm{T}_{\mathrm{s}}=0$ ) becomes.

$$
\Delta T_{w}^{\prime}=\frac{\Delta T_{f} B i_{m}}{B i_{m}+1}
$$

where $B i_{m}$ is the ratio of thermal resistance of the composite solid mesh cell to that of the fluid:

$$
B i_{m}=\left(\frac{\Delta x_{s}}{k_{s}}+\frac{\Delta x_{T B C}}{k_{T B C}}\right)\left(\frac{k_{f}}{\Delta x_{f}}\right)
$$

The correct target wall temperature response $\Delta \mathrm{T}_{\mathrm{w}}$ on the other hand can only be obtained if the solid mesh point is within the TBC layer with a mesh spacing equal to the penetration depth $\delta_{p}$ :

$$
\Delta T_{w}=\frac{\Delta T_{f} B i_{\delta p}}{B i_{\delta p}+1}
$$

$B i_{\delta_{p}}$ is the ratio of solid mesh thermal resistance when the mesh spacing is equal to the penetration depth, to that of the fluid:

$$
B i_{\delta_{p}}=\left(\frac{\delta_{p}}{k_{T B C}}\right)\left(\frac{k_{f}}{\Delta x_{f}}\right)
$$

Thus, the ratio of the erroneously calculated wall temperature response $\Delta T_{w}^{\prime}$ to the targeted correct one $\Delta T_{w}$ is, 


$$
\frac{\Delta T_{w}^{\prime}}{\Delta T_{w}}=\frac{B i_{m}}{B i_{\delta_{p}}} \frac{\left(1+B i_{\delta_{p}}\right)}{\left(1+B i_{m}\right)}
$$

The corresponding ratio of the erroneously calculated wall heat flux response $\Delta \mathrm{q}^{\prime}$ to the targeted correct value $\Delta \mathrm{q}$ becomes:

$$
\frac{\Delta q^{\prime}}{\Delta q}=\frac{\left(1+B i_{\delta_{p}}\right)}{\left(1+B i_{m}\right)}
$$

To make use of the interface response analysis, we need to know relevant frequencies to determine the corresponding penetration depths. A near-wall turbulence velocity spectrum (shown in Fig.15) indicates that the frequency range with high unsteady fluctuations is between $1000 \mathrm{~Hz}$ and $100,000 \mathrm{~Hz}$. Given the local mesh spacing for the fluid and solid sides and the frequency range of interest, we can analytically evaluate the errors in unsteady wall temperature response and heat flux response at selected frequencies by using Eqs. 39, 40. The corresponding sample analytical results for point A are shown in Table 1. Also listed in Table 1 are the corresponding results of the single-layer without TBC (taking $\Delta x_{T B C}=0$, and $k_{T B C}=k_{S}$ in Eqs 36 and 38).

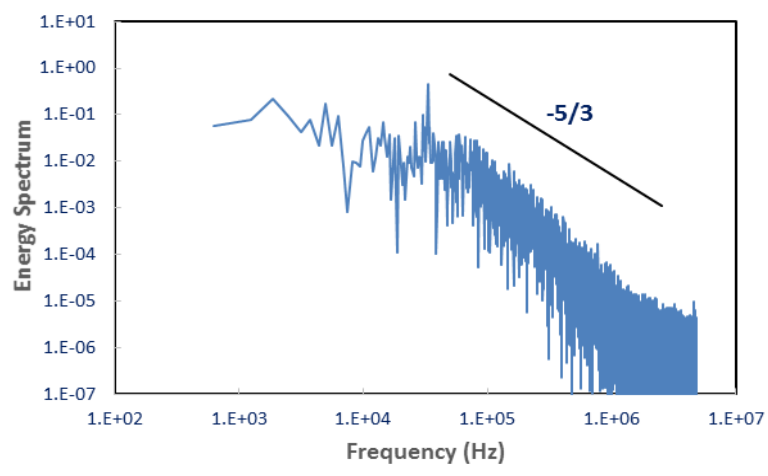

Fig.15, Calculated Velocity Power Spectrum near Blade Trailing Edge.

\begin{tabular}{|l|l|c|c|c|c|}
\hline \multicolumn{2}{|c|}{ Frequency } & $\mathbf{5 , 0 0 0 H z}$ & $\mathbf{1 0 , 0 0 0 H z}$ & $\mathbf{5 0 , 0 0 0 H z}$ & $\mathbf{1 0 0 , 0 0 0 H z}$ \\
\hline \multirow{2}{*}{$\delta_{\boldsymbol{p}} / \Delta \boldsymbol{x}_{\boldsymbol{s}}$} & single layer & 0.992 & 0.701 & 0.314 & 0.221 \\
\cline { 2 - 6 } & with TBC & 0.067 & 0.047 & 0.021 & 0.015 \\
\hline$\frac{\Delta \boldsymbol{T}_{\boldsymbol{w}}^{\prime}}{\Delta \boldsymbol{T}_{\boldsymbol{w}}}$ & single layer & 1.01 & 1.41 & 3.30 & 4.44 \\
\cline { 2 - 6 } & with TBC & 2.36 & 3.13 & 5.75 & 7.80 \\
\hline$\frac{\Delta \boldsymbol{q}^{\prime}}{\Delta \boldsymbol{q}}$ & single layer & 0.999 & 0.995 & 0.989 & 0.988 \\
\cline { 2 - 6 } & with TBC & 0.30 & 0.268 & 0.229 & 0.220 \\
\hline
\end{tabular}

Table 1: Ratios of erroneous $\mathrm{T}_{\mathrm{w}}$ and $\mathrm{q}$ responses to target values at different frequencies (Analytical results from the interface response analysis).

For the TBC case, the low thermal conductivity leads to a low penetration depth, thus a much higher unsteady wall temperature response. Hence the true physical wall temperature fluctuations should be much higher than those in the case without TBC. The contrast in the wall 
temperature unsteadiness between the two cases with and without TBC is consistently shown by both the analytical results (Table 1) and the LES-CHT solutions (Fig.12 vs Fig.10). Both the analytic and computational results also consistently show that the errors of the directly-coupled interface method are much higher in the TBC case than the single layer no-TBC case. Consequently, we see much more significant errors in over-predicted unsteady wall temperatures and under-predicted unsteady heat fluxes for the TBC case. More specifically, the simple interface response analysis (Table 1) shows an over-prediction of wall temperature fluctuations by a factor about 5-8, and an under-prediction of the wall heat flux by a factor of about 3-4. They are in qualitative agreement with the LES-CHT (Figs.10-13) solutions.

The frequency-dependent analytical results of the interface response analysis provide new insight into characteristics of the extra solid-mesh dependency. The consistent comparisons between the analytical and the LES-CHT results also suggest that the simple interface response analysis may usefully serve to indicate magnitudes of errors in unsteady wall temperature and heat flux predictions, should a directly-coupled interface method have to be used.

Overall, the computational results underpinned by the simple analytical results underline the advantages of the present moving-average based interface method over the conventional directly coupled method, particularly in the context of CHT solutions with turbulence scaleresolved flow solvers.

\section{Concluding Remarks}

In the present work, a moving-average based method is developed for monolithic CHT solutions where both the fluid and solid domains are solved using the same solver and are closely coupled, equivalently to a simple Dirichlet-Dirichlet interface condition. Recognising the huge time-scale disparity between fluid and solid, the primary approach adopted is to convert an unsteady time-domain presentation of the solid conduction to its frequency domain counterpart, thus enabling for the solid domain to be very efficiently solved in a steady-like solution method. Effectively the solid side is coupled with the timeaveraged fluid side. In addition, wall temperature unsteadiness can be included efficiently in the interface coupling by the corresponding semi-analytical harmonic transfer functions, supported by a Fourier Transform of the near-wall fluid temperatures on-the-fly. As such, the unsteady conduction effects on the fluid has been included effectively and accurately without even numerically solving an unsteady conduction field in the solid domain.

An illustrative analysis for the conditions considered in the present study indicates that a stability for such moving-average interface coupling should require no more stringent conditions than those for stable fluid and solid domain solutions. A stable behaviour of the moving-average interface treatment has been shown in all the present numerical test cases.

The present computational results highlight that the use of a conventional timesynchronised directly coupled interface can lead to significant extra mesh dependence in the solid domain, manifested as over-predicted wall temperature fluctuations and underpredicted heat flux fluctuations. The proposed moving-average based interface method however is very effective in reducing these errors. The present computational results, aided by a new two-layer interface response analysis, also illustrate a much augmented sensitivity of unsteady wall temperatures and heat fluxes to the interface treatment, when a Thermal Barrier Coating (TBC) layer of low conductivity is introduced. 
Appendix, Surface Temperature-Flux Transfer Function for Semi-Infinite Solid Domain.

Unsteady heat transfer at a high frequency into blade surface covered by a Thermal Barrier Coating (TBC) can be approximated as a 1D semi-infinite solid domain covered by a thin solid layer (thickness $\lambda$ ) of low thermal conductivity (as shown in Fig.A1).

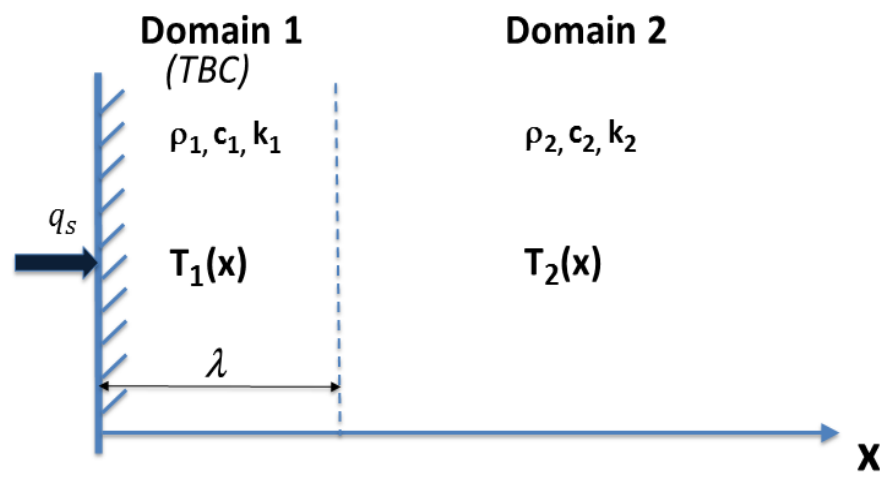

Fig.A1, 1-D configuration of TBC layer on semi-infinite solid domain

With given constant material properties: density $\rho$, specific heat c, thermal conductivity $\mathrm{k}$, and thermal diffusivity $\alpha\left(\frac{k}{\rho c}\right)$, the conduction equations for the two domains are:

$\begin{array}{lll}\text { TBC layer: } & \frac{\partial T_{1}}{\partial t}=\alpha_{1} \frac{\partial^{2} T_{1}}{\partial x^{2}} \\ \text { Semi-infinite domain: } & \frac{\partial T_{2}}{\partial t}=\alpha_{2} \frac{\partial^{2} T_{2}}{\partial x^{2}}\end{array}$

The wall surface $(\mathrm{x}=0)$ is subject to an unsteady heat flux at frequency $\omega$ :

$$
q_{s}=\tilde{q}_{s}+\operatorname{real}\left(\hat{q}_{s} \mathrm{e}^{i \omega t}\right) \text {. }
$$

The corresponding unsteady temperatures within the solid domains can also be expressed in a harmonic form:

$$
T(x, t)=\tilde{T}(x)+\operatorname{real}\left(\widehat{T} \mathrm{e}^{i \omega t}\right) .
$$

In the reference by Schultz, D.L., and Jones (1973), Eqs A1 and A2 were solved by first taking a Laplace transform, and the resultant 2nd order ODEs were then solved analytically leading to spatial $\mathrm{T}$ distributions in the Laplace space. As such the temperature distributions in the physical time domain would have to be obtained through inverting the Laplace transform. In the present work, we would like to get the T distributions in the frequency domain directly without involving a transform. Keeping in mind that the Laplace transform was introduced primarily for converting the PDEs to ODEs, we then find that the frequency domain formulation of a harmonic unsteadiness will simply enable us to do just that.

For domain 1, substitute the harmonic expression for the temperature in Eq.A1, it becomes:

$$
\operatorname{Real}\left(i \omega \widehat{T}_{1} \mathrm{e}^{i \omega t}\right)=\alpha_{1}\left[\frac{\partial^{2} \widetilde{T}_{1}}{\partial x^{2}}+\operatorname{Real}\left(\frac{\partial^{2} \widehat{T}_{1}}{\partial x^{2}} \mathrm{e}^{i \omega t}\right)\right]
$$


Noting a linear temperature variation for the 1D steady conduction, we thus have for the steady component, $\frac{\partial^{2} \widetilde{T}_{1}}{\partial x^{2}}=0$. The resultant harmonically balanced equation for the temperature harmonic then becomes a $2^{\text {nd }}$ order ODE:

$$
\frac{d^{2} \widehat{T}_{1}}{d x^{2}}-\frac{i \omega}{\alpha_{1}} \widehat{T}_{1}=0
$$

In the same way, we have for domain 2:

$$
\frac{d^{2} \widehat{T}_{2}}{d x^{2}}-\frac{i \omega}{\alpha_{2}} \widehat{T}_{2}=0
$$

The rest of the procedure for the solution of temperature harmonic distributions will be similar to that of Schultz and Jones (1973). The main difference is that the present temperatures are harmonics in frequency domain instead of the Laplace transformed ones.

For the $2^{\text {nd }}$ order ODEs (Eq.A6), standard general solutions are:

$$
\begin{aligned}
& \widehat{T}_{1}=C_{1} e^{x\left(\frac{i \omega}{\alpha_{1}}\right)^{1 / 2}}+C_{2} e^{-x\left(\frac{i \omega}{\alpha_{1}}\right)^{1 / 2}} \\
& \widehat{T}_{2}=C_{3} e^{x\left(\frac{i \omega}{\alpha_{2}}\right)^{1 / 2}}+C_{4} e^{-x\left(\frac{i \omega}{\alpha_{2}}\right)^{1 / 2}}
\end{aligned}
$$

The four constants can be fixed by making use of the four boundary conditions:

$$
\begin{array}{ll}
\text { At wall surface }(\mathrm{x}=0): & -k_{1} \frac{\partial \widehat{T}_{1}}{\partial x}=\hat{q}_{s} ; \\
\text { At interface between the two domains }(\mathrm{x}=\lambda): & k_{1} \frac{\partial \widehat{T}_{1}}{\partial x}=k_{2} \frac{\partial \widehat{T}_{2}}{\partial x} ; \\
& \frac{\partial \widehat{T}_{2}}{\partial x}=0, \quad \text { and } \widehat{T}_{2}=0 .
\end{array}
$$

Consequently, the following harmonic temperature distributions in the two domains are obtained:

$$
\begin{aligned}
& \widehat{T}_{1}=\frac{\hat{q}_{s} \sqrt{\alpha_{1}}}{k_{1} \sqrt{i \omega}}\left[\frac{(1+a) e^{-(x-\lambda)\left(\frac{i \omega}{\alpha_{1}}\right)^{\frac{1}{2}}}+(1-a) e^{(x-\lambda)\left(\frac{i \omega}{\alpha_{1}}\right)^{\frac{1}{2}}}}{\left.(1+a) e^{\lambda\left(\frac{i \omega}{\alpha_{1}}\right)^{\frac{1}{2}}}-(1-a) e^{-\lambda\left(\frac{i \omega}{\alpha_{1}}\right)^{\frac{1}{2}}}\right]}\right. \\
& \widehat{T}_{2}=\frac{2 \hat{q}_{s} \sqrt{\alpha_{1}}}{k_{1} \sqrt{i \omega}}\left[\frac{e^{(\lambda-x)\left(\frac{i \omega}{\alpha_{2}}\right)^{\frac{1}{2}}}}{\left.(1+a) e^{\lambda\left(\frac{i \omega}{\alpha_{1}}\right)^{\frac{1}{2}}}-(1-a) e^{-\lambda\left(\frac{i \omega}{\alpha_{1}}\right)^{\frac{1}{2}}}\right]}\right.
\end{aligned}
$$

where constant $a$ is given by the ratios of the materail properties, $a=\sqrt{\frac{\rho_{2} c_{2} k_{2}}{\rho_{1} c_{1} k_{1}}}$.

Now, return to the main aim to establish the harmonic temperature-flux transfer function at the solid surface with a TBC layer of thickness $\delta$ for a given frequency $\omega$.

$$
\hat{q}_{s}=\hat{C}_{T q} \widehat{T}_{s}
$$

For a case with a TBC layer of thickness $\lambda$, this is obtained by setting $\mathrm{x}=0$ in Eq.A8a.

$$
\left(\hat{C}_{T q}\right)_{T B C}=\frac{k_{1} \sqrt{i \omega}}{\sqrt{\alpha_{1}}}\left[\frac{(1+a) e^{\lambda\left(\frac{i \omega}{\alpha_{1}}\right)^{\frac{1}{2}}}-(1-a) e^{-\lambda\left(\frac{i \omega}{\alpha_{1}}\right)^{\frac{1}{2}}}}{\left.(1+a) e^{\lambda\left(\frac{i \omega}{\alpha_{1}}\right)^{\frac{1}{2}}}+(1-a) e^{-\lambda\left(\frac{i \omega}{\alpha_{1}}\right)^{\frac{1}{2}}}\right]}\right.
$$

For a case without a TBC layer, domain 1 and domain 2 have the same material $(a=1)$. The corresponding T-q transfer function for a single layer without TBC then is simply:

$$
\left(\hat{C}_{T q}\right)_{\text {without TBC }}=\sqrt{i \omega} \sqrt{\rho c k}
$$




\section{References}

Cada, M. and Torrilhon, M., 2009, Compact Third-order Limiter Functions for Finite Volume Methods, J. of Computational. Physics, Vol.228(11), pp4118-4145.

Duchaine, F., Corpron, A., Pons, L., Moureau, V., Nicoud, F., and Poinsot, T., 2009, Development and Assessment of a Coupled Strategy for Conjugate Heat Transfer with Large Eddy Simulation: Application to a Cooled Turbine Blade, Int. J. Heat Fluid Flow, 30(6), pp. 1129-1141.

Errera M, and Baqué B. 2013, A Quasi-dynamic Procedure for Coupled Thermal Simulations, International Journal for Numerical Methods in Fluids, Vol.72, pp11831206.

Fadl, M., He, L., 2017, On Large Eddy Simulation based Conjugate Heat Transfer Procedure for Transient Natural Convection, Journal of Turbomachinery, Vol.139, Issue 11.

Faghri, A., Zhang, Y., and Howell, J. R., 2010, Advanced Heat and Mass Transfer, Global Digital Press, Columbia, MO.

Fawcett, R. J., Wheeler, A. S., He, L., and Taylor, R., 2013, Experimental Investigation into the Impact of Crossflow on the Coherent Unsteadiness within Film Cooling Flows. International Journal of Heat and Fluid Flow, Vol. 40, April 2013.

Gerolymos, G.A. Michon, G.J., Neubauer, J., 2002, Analysis and Application of Chorochronic Periodicity in Turbomachinery Rotor/Stator Interaction Computations. Journal of Propulsion and Power, Vol.18, No.5, pp. 1139-1152.

Giles, M.B., 1997, Stability Analysis of Numerical Interface Conditions in Fluid-structure Thermal Analysis, International Journal for Numerical Methods in Fluids, Vol.25, No.4, pp421-436.

He, L., 1992, Method of Simulating Unsteady Turbomachinery Flows with Multiple Perturbations, AIAA Journal, Vol.30, No.11, pp 2730-2735.

He, L., 1993, New Two-Grid Acceleration Method for Unsteady Navier-Stokes Calculations, AIAA Journal of Propulsion and Power, Vol.9, No.2, pp.272-280,.

He, L. and Oldfield, M.L.G., 2011, Unsteady Conjugate Heat Transfer Modelling, Journal of Turbomachinery, 2011, Vo1.133, No.3.

He, L., 2008, Harmonic Solution of Unsteady Flow around Blade with Separation, AIAA Journal, Vol.46, No.6.

He, L., 2013, Fourier Spectral Method for Multi-scale Aerothermal Analysis, International Journal of Computational Fluid Dynamics, Vol.27, No.2, pp118-129.

He, L. and Fadl, M., 2016, Multi-scale Time Integration for Transient Conjugate Heat Transfer, International Journal for Numerical Methods in Fluids, Vol.83, Issue 12, pp887-904, (DOI: 10.1002/fld.4295), online publication Sept, 2016.

He, L., 2017, Multiscale Block Spectral Solution for Unsteady Flows, International Journal for Numerical Methods in Fluids, Vol.86, Issue 10, pp655-678. 
Hylton, L.D., Mihelc, M.S. Turner, E.R. Nealy, D.A. and York. R.E. 1983, Analytical and Experimental Evaluation of the Heat Transfer Distribution over the Surface of Turbine Vanes. NASA Technical Report, CR 168015.

Jaure, S., Duchaine F., Staffelbach, G., and Gicquel, L.Y.M, 2013, Massively parallel Conjugate Heat Transfer Methods relying on Large Eddy Simulation applied to an Aeronautical Combustor, Computational Science \& Discovery 6 (2013) 015008,.

Koren, C., Vicquelin, R. and Gicquel, O., 2018, Multiphysics Simulation Combining Large-Eddy Simulation, Wall Heat Conduction and Radiative Energy Transfer to Predict Wall Temperature Induced by a Confined Premixed Swirling Flame, Flow, Turbulence and Combustion, Vol.101(1), pp77-108.

Moffat, R. J., 1998, What's New in Convective Heat Transfer? International Journal of Heat and Fluid Flow, Vol.19, pp90-101.

Maffulli, R., and He, L., 2014, Wall Temperature Effects on Heat Transfer Coefficient for High Pressure Turbines, Journal of Propulsion and Power, 2014, Vol.30, No. 4, pp.1080-1090.

Maffulli, R., and He, L., 2017, Impact of Wall Temperature on Heat Transfer Coefficient and Aerodynamics for Three-Dimensional Turbine Blade Passage, J. Thermal Sci. Eng. Appl, Vol. 9, No. 4.

Liou, M-S, 2006, A Sequel to AUSM, Part II: AUSM+-up for all speeds, An Accurate and Robust Flux Splitting Scheme for Shock and Contact Discontinuities, J. of Computational Physics, Vol. 214 (1), pp.137-170, 2006.

Perelman, T.L., 1961, On Conjugated Problems of Heat Transfer, International Journal of Heat and Mass Transfer, Vol.3. No.4, pp293-303, 1961.

Shahi, M., Kok, J. B. W., Roman Casado, J. C., and Pozarlik, A. K., 2014, Study of Unsteady Heat Transfer as a Key Parameter to Characterize Limit Cycle of High Amplitude Pressure Oscillations, ASME Paper GT2014-26311.

Schultz, D.L., and Jones, T.V., 1973, Heat Transfer Measurement in Short Duration Facilities, AGARD AG-165.

Sun Z, Chew J.W., Hills N., Volkov K. and Barnes C., 2010, Efficient Finite Element Analysis/Computational Fluid Dynamics Thermal Coupling for Engineering Applications, Journal of Turbomachinery, Vo1.132, No.3.

Tiselj, I., Oder J, and Cizelj L. 2013, Double-sided Cooling of Heated slab: Conjugate Heat Transfer DNS, International Journal of Heat and Mass Transfer, 66(2013), 781-790.

Tyagi, M., Acharya, S., 2003. Large Eddy Simulation of Film Cooling Flow from an Inclined Cylindrical Jet. Journal of Turbomachinery 125 (4), 734-742, 2003.

Verstraete, T., and Scholl, S., 2016, Stability Analysis of Partitioned Methods for Predicting Conjugate Heat Transfer, International Journal of Heat and Mass Transfer, Vol.101, pp852-869.

Zhang, Q., and He, L., 2014, Impact of Wall Temperature on Turbine Blade Tip AeroThermal Performance, J. Eng. Gas Turb. and Power, Vol.136, No.5, 052602. 